\title{
LARGE GLOBAL SOLUTIONS FOR NONLINEAR SCHRÖDINGER EQUATIONS I, MASS-SUBCRITICAL CASES
}

\author{
MARIUS BECEANU, QINGQUAN DENG, AVY SOFFER, AND YIFEI WU
}

\begin{abstract}
In this paper, we consider the nonlinear Schrödinger equation,

$$
i \partial_{t} u+\Delta u=\mu|u|^{p} u, \quad(t, x) \in \mathbb{R}^{d+1},
$$

with $\mu= \pm 1, p>0$.

In this work, we consider the mass-subcritical cases, that is, $p \in\left(0, \frac{4}{d}\right)$. We prove that under some restrictions on $d, p$, any radial initial data in the critical space $\dot{H}^{s_{c}}\left(\mathbb{R}^{d}\right)$ with compact support, implies global well-posedness.
\end{abstract}

\section{CONTENTs}

1. Introduction 2

2. Preliminary 5

2.1. Notation 5

2.2. Some basic lemmas 6

2.3. Linear Schrödinger operator 8

3. Linear flow estimates on localized functions 11

4. Nonlinear flow estimates on localized initial data 18

4.1. Local theory 18

4.2. Nonlinear estimates on $v \quad 21$

5. The proof of Theorem 1.1

5.1. Nonlinear estimates on $w$

5.2. Global existence 31

Acknowledgements 32

References 33

2010 Mathematics Subject Classification. Primary 35Q55.

Key words and phrases. Nonlinear Schrödinger equation, global well-posedness, critical regularity. 


\section{INTRODUCTION}

We study the Cauchy problem for the following nonlinear Schrödinger equation (NLS) on $\mathbb{R} \times \mathbb{R}^{d}$ :

$$
\left\{\begin{array}{l}
i \partial_{t} u+\Delta u=\mu|u|^{p} u, \\
u(0, x)=u_{0}(x)
\end{array}\right.
$$

with $\mu= \pm 1, p>0$. Here $u(t, x): \mathbb{R} \times \mathbb{R}^{d} \rightarrow \mathbb{C}$ is a complex-valued function. The case $\mu=1$ is referred to the defocusing case, and the case $\mu=-1$ is the focusing case. The class of solutions to equation (1.1) is invariant under the scaling

$$
u(t, x) \rightarrow u_{\lambda}(t, x)=\lambda^{\frac{2}{p}} u\left(\lambda^{2} t, \lambda x\right) \text { for } \lambda>0,
$$

which maps the initial data as

$$
u(0) \rightarrow u_{\lambda}(0):=\lambda^{\frac{2}{p}} u_{0}(\lambda x) \text { for } \lambda>0 .
$$

Denote

$$
s_{c}=\frac{d}{2}-\frac{2}{p} .
$$

Then the scaling leaves $\dot{H}^{s_{c}}$ norm invariant, that is,

$$
\|u\|_{\dot{H}^{s_{c}}}=\left\|u_{\lambda}\right\|_{\dot{H}^{s_{c}}},
$$

which is called critical regularity $s_{c}$. It is also considered as the lowest regularity for which the problem (1.1) is well-posed for general $H^{s}\left(\mathbb{R}^{d}\right)$-data. Indeed, it was proved by Christ, Colliander, Tao [12] that there exist some initial datum belonging to $H^{s}\left(\mathbb{R}^{d}\right), s<s_{c}$ such that the problem (1.1) is ill-posed.

The $H^{1}$-solution of equation (1.1) also enjoys mass, momentum and energy conservation laws, which read

$$
\begin{aligned}
M(u(t)) & :=\int|u(t, x)|^{2} d x=M\left(u_{0}\right), \\
P(u(t)) & :=\operatorname{Im} \int \overline{u(t, x)} \nabla u(t, x) d x=P\left(u_{0}\right), \\
E(u(t)) & :=\int|\nabla u(t, x)|^{2} d x+\frac{2 \mu}{p+2} \int|u(t, x)|^{p+2} d x=E\left(u_{0}\right) .
\end{aligned}
$$

The well-posedness and scattering theory for Cauchy problem (1.1) with initial data in $H^{s}\left(\mathbb{R}^{d}\right)$ were extensively studied, which we here briefly review. The local well-posedness theory follows from a standard fixed point argument, implying that for all $u_{0} \in H^{s}\left(\mathbb{R}^{d}\right)$, there exists $T_{0}>0$ such that its corresponding solution $u \in C\left(\left[0, T_{0}\right), H^{s}\left(\mathbb{R}^{d}\right)\right)$. In fact, the above $T_{0}$ depends on $\left\|u_{0}\right\|_{H^{s}\left(\mathbb{R}^{d}\right)}$ when $s>s_{c}$ and also the profile of $u_{0}$ when $s=s_{c}$. Some of the results can be found in Cazenave and Weissler [10].

Such argument can be applied directly to prove the global well-posedness for solutions to equation (1.1) with small initial data in $H^{s}\left(\mathbb{R}^{d}\right)$ with $s \geq s_{c}$. In the mass-subcritical cases, that is, $p<\frac{4}{d}$, if we consider the solution in $L^{2}\left(\mathbb{R}^{d}\right)$ space, the local theory above, together with the mass conservation laws (1.3), yields the global well-posedness for any initial data $u_{0} \in L^{2}\left(\mathbb{R}^{d}\right)$. In the mass-supercritical, energy-subcritical cases, that is, $\frac{4}{d}<p<\frac{4}{d-2}$, if we consider the solution in energy space $H^{1}\left(\mathbb{R}^{d}\right)$, the local theory above together with conservation laws (1.3) yields the global well-posedness for all initial data $u_{0} \in H^{1}\left(\mathbb{R}^{d}\right)$ in 
the defocusing case $\mu=1$, and for any initial data $u_{0} \in H^{1}\left(\mathbb{R}^{d}\right)$ with some restrictions in the focusing case. Furthermore, the scattering under the same conditions were also obtained by Ginibre, Velo [27] in the defocusing case and [24] in the focusing case. In the mass-critical and energy-critical cases, since the conservation laws do not imply directly the global existence of the solutions, the problem becomes much more complicated. In the energy-critical case, the global well-posedenss and scattering in the defocusing case was first proved by Bourgain [3] in the radial data case and then by Colliander, Keel, Takaoka, Staffilani and Tao [13] in the non-radial data case in dimension three, the higher dimension cases were solved by Ryckman and Visan [59] and Visan [65, 66]; the global well-posedenss and scattering in the focusing case was proved by Kenig and Merle [30] in the radial data case, then by Killip, Visan [42] in the non-radial case when the dimensions are five and higher, and by Dodson [21] in four dimensions, see also [43, 61, 62, 65, 66] for some previous works and simplified proofs. In the mass-critical case, the global well-posedenss and scattering was first proved by Killip, Tao, Visan [39] in the radial data case in dimension two, and Killip, Visan, Zhang [45] in dimensions higher than two, then in the non-radial data case, the problem was solved in a series of papers of Dodson [17, 18, 19, 20].

More complicated situation appears if one considers the general nonlinear Schrödinger equations in the critical space $\dot{H}^{s_{c}}\left(\mathbb{R}^{d}\right)$. Recently, conditional global and scattering results with the assumption of $u \in L_{t}^{\infty}\left(I, \dot{H}_{x}^{s_{c}}\left(\mathbb{R}^{d}\right)\right)$ (here $I$ is the maximal lifespan) were considered by many authors, which was started from [31, 32, and then developed by [5, 23, 25, 26, 33, 36, 37, 40, 41, 42, 48, 49, 50, 51, 67] and cited references. That is, if the initial data $u_{0} \in \dot{H}^{s_{c}}\left(\mathbb{R}^{d}\right)$ and the solution has priori estimate

$$
\sup _{0<t<T_{\text {out }}\left(u_{0}\right)}\|u\|_{\dot{H}_{x}^{s c}\left(\mathbb{R}^{d}\right)}<+\infty
$$

then $T_{\text {out }}\left(u_{0}\right)=+\infty$ and the solution scatters in $\dot{H}^{s_{c}}\left(\mathbb{R}^{d}\right)$, here $\left[0, T_{\text {out }}\left(u_{0}\right)\right)$ is the maximal interval in positive direction for existence of the solution. Consequently, these results give the blowup criterion which the lifetime depends only on the critical norm $\|u\|_{L_{t}^{\infty} \dot{H}_{x}^{s c}\left(I \times \mathbb{R}^{d}\right)}$. However, it seems that no such large data global results are known, if only the initial data $u_{0} \in \dot{H}^{s_{c}}\left(\mathbb{R}^{d}\right)$. Furthermore, many authors considered the large global solutions for rough data from a probabilistic point of view, that is, one may construct a large sets of initial data of super-critical regularity which leads to global solutions, see [1, 2, 6, 7, 8, 14, 15, 16, 22, 38, 52, 53, 54, 55, 56, 57, 58, 63].

In the first part of our series of works, we consider the global solution for the masssubcritical nonlinear Schödinger equation in the critical space $\dot{H}^{s_{c}}\left(\mathbb{R}^{d}\right)$. Due to the mass conservation law, $L^{2}$-initial datum lead to the global solutions. It is known from Christ, Colliander and Tao [12 and Kenig, Ponce, Vega [35] that the problem is ill-posed in some sense for the non-radial datum in $\dot{H}^{s}\left(\mathbb{R}^{d}\right), s<0$. However, for the radial data, due to the better radial Strichartz estimates, one may establish the local well-posedness result in negative regularity Sobolev spaces. Indeed, it was proved by Guo and Wang [28] that there exists $p_{0}(d)<\frac{4}{d}$, such that for any $p \in\left(p_{0}(d), \frac{4}{d}\right)$, if the initial datum are radial and small in the critical space $\dot{H}^{s_{c}}\left(\mathbb{R}^{d}\right)$, then the nonlinear solutions of (1.1) are global and scatter. Very recently, Killip, Masaki, Murphy and Visan [36, 37] proved a conditional result; that in the defocusing case, there exists $p_{0}(d)<\frac{4}{d}$, such that for any $p \in\left(p_{0}(d), \frac{4}{d}\right)$, if the radial 
solution $u \in L_{t}^{\infty} \dot{H}_{x}^{s_{c}}\left(I \times \mathbb{R}^{d}\right)$, then $I=\mathbb{R}$ and the solution scatters, by using concentrationcompactness arguments. This is the first global result for large data theory in the critical spaces for the mass-subcritical NLS.

In this paper, we prove unconditional global well-posedness. We prove that for radial initial data with compact support in space, and is in the critical space, there exists solution global in time.

Theorem 1.1. Let $d \geq 4$, and $\mu= \pm 1$. Then there exists $p_{0}(d) \in\left(0, \frac{4}{d}\right)$, such that for any $p \in\left[p_{0}(d), \frac{4}{d}\right)$, the following is true. Suppose that $u_{0} \in \dot{H}^{s_{c}}\left(\mathbb{R}^{d}\right)$ is a radial function satisfying

$$
\text { supp } u_{0} \subset\{x:|x| \leq 1\} .
$$

Then the solution $u$ to the equation (1.1) with the initial data $u_{0}$ exists globally in time, and $u \in C\left(\mathbb{R}^{+} ; \dot{H}^{s_{c}}\left(\mathbb{R}^{d}\right)\right) \cap L^{\infty}\left(\mathbb{R}^{+} ; \dot{H}^{s_{c}}\left(\mathbb{R}^{d}\right)+L^{2}\left(\mathbb{R}^{d}\right)\right)$. Moreover, for any $t \in \mathbb{R}$,

$$
\|u(t)\|_{\dot{H}^{s_{c}\left(\mathbb{R}^{d}\right)}} \lesssim 1+|t| .
$$

Remark 1.2. We make several remarks regarding the above statements.

(1) Our conclusions are valid for both of the focusing and the defocusing cases. Further, by scaling, one can extend the size of the radius 1 to an arbitrary large number. Moreover, the compact support assumption on initial data are not necessary and can be replaced by some weighted assumption.

(2) In the present paper, we are not going to give the sharp conditions on $p_{0}(d)$ and $d$.

In the mass-subcritical cases, there is a new difficulty when we consider the global solution in the negative Sobolev space. It is worth noting that in this case, we can not use the mass, energy conservation laws, and Morawetz estimates. Moreover, the pseudo-conformal conservation law has no good sign.

Further, because all of the conservation laws are beyond the critical scaling regularity, we believe that analogous scattering result in $\dot{H}^{s_{c}}\left(\mathbb{R}^{d}\right)$ is very hard to pursue in the masssubcritical case (it is similar to the energy-supercritical case in which all the conservation laws are below the critical scaling regularity), even if the initial data is smooth enough.

\section{Sketch of the proof:}

First, in step 1, we show an improved (supercritical) Strichartz estimates for the initial data localized in space under the linear flow. More precisely, we prove that for all $N \geq 1$, there exist $\alpha_{0}>1, \beta_{0}>0$, such that

$$
\left\|\left\langle t^{\alpha_{0}}|\nabla|\right\rangle^{\beta_{0}}|\nabla|^{s_{c}}\left(e^{i t \Delta} \chi_{\leq 10}\left(P_{\geq N} g\right)\right)\right\|_{L_{t}^{2} L_{x}^{\frac{2 d}{d-2}}\left(\mathbb{R}^{\left.\frac{1}{d} \mathbb{R}^{d}\right)}\right.} \lesssim\left\|P_{\geq N} g\right\|_{\dot{H}^{s_{c}}\left(\mathbb{R}^{d}\right)}
$$

(a slight stronger estimate is needed, see Section 5 below). From this estimate, we gain the regularity and time decay for $t \gtrsim 1$.

In step 2 , given small constant $\delta_{0}>0$, we break the initial data into two parts, $u_{0}=$ $v_{0}+w_{0}$, with

$$
v_{0}=\chi_{\leq 10}\left(P_{\geq N} u_{0}\right) \text { with }\left\|v_{0}\right\|_{\dot{H}^{s}\left(\mathbb{R}^{d}\right)} \leq \delta_{0}, \quad \text { and } \quad w_{0} \in L^{2}\left(\mathbb{R}^{d}\right) .
$$

Now, let $v$ be the solution of the following time cut-off equation,

$$
\left\{\begin{array}{l}
i \partial_{t} v+\Delta v=\chi_{\leq 1}(t)|v|^{p} v \\
v(0, x)=v_{0}(x)
\end{array}\right.
$$


In this step, we prove that the analogous estimates in Step 1 hold true for the nonlinear solution $v$. That is,

$$
\left\|\left\langle t^{\alpha_{0}}|\nabla|\right\rangle^{\beta_{0}}|\nabla|^{s_{c}} P_{M} v\right\|_{l_{M}^{\infty} L_{t}^{2} L_{x}^{\frac{2 d}{d-2}}\left(\{M \geq 1\} \times \mathbb{R} \times \mathbb{R}^{d}\right)} \lesssim\left\|v_{0}\right\|_{\dot{H}^{s_{c}}\left(\mathbb{R}^{d}\right)}
$$

which we use later with $t \gtrsim 1$.

In step 3, we prove the uniform in time boundedness of $\|w(t)\|_{L_{x}^{2}\left(\mathbb{R}^{d}\right)}$. Note that $w$ obeys the equation of

$$
i \partial_{t} w+\Delta w=|u|^{p} u-\chi_{\leq 1}(t)|v|^{p} v
$$

We find that the nonlinearity obeys

$$
\left.|| u\right|^{p} u-\chi_{\leq 1}(t)|v|^{p} v \mid \lesssim\left(|u|^{p}+\left|\chi_{\lesssim 1}(t) v\right|^{p}\right)\left(|w|+\left|\chi_{\gtrsim 1}(t) v\right|\right) .
$$

Due to the good estimates on $\chi_{\gtrsim 1}(t) v$ obtained in Step 2, we can prove the desired estimate by the almost mass conservation of $w$.

\section{Preliminary}

2.1. Notation. We write $X \lesssim Y$ or $Y \gtrsim X$ to indicate $X \leq C Y$ for some constant $C>0$. If $C$ depends upon some additional parameters, we will indicate this with subscripts; for example, $X \lesssim a Y$ denotes the assertion that $X \leq C(a) Y$ for some $C(a)$ depending on $a$. We use $O(Y)$ to denote any quantity $X$ such that $|X| \lesssim Y$. We use the notation $X \sim Y$ whenever $X \lesssim Y \lesssim X$. Moreover, we use the notation $X \ll Y$ to indicate $X \leq C^{-1} Y$.

The notation $|\nabla|^{\alpha}=\left(-\partial_{x}^{2}\right)^{\alpha / 2}$. We denote $\mathcal{S}\left(\mathbb{R}^{d}\right)$ to be the Schwartz Space in $\mathbb{R}^{d}$, and $\mathcal{S}^{\prime}\left(\mathbb{R}^{d}\right)$ to be the topological dual of $\mathcal{S}\left(\mathbb{R}^{d}\right)$. Let $h \in \mathcal{S}^{\prime}\left(\mathbb{R}^{d+1}\right)$, we use $\|h\|_{L_{t}^{q} L_{x}^{p}}$ to denote the mixed norm $\left(\int\|h(\cdot, t)\|_{L^{p}}^{q} d t\right)^{\frac{1}{q}}$, and $\|h\|_{L_{x t}^{q}}:=\|h\|_{L_{x}^{q} L_{t}^{q}}$. Sometimes, we use the notation $q^{\prime}=\frac{q}{q-1}$.

Throughout this paper, we use $\chi_{\leq a}$ for $a \in \mathbb{R}^{+}$to be the smooth function

$$
\chi_{\leq a}(x)=\left\{\begin{array}{l}
1,|x| \leq a \\
0,|x| \geq \frac{11}{10} a .
\end{array}\right.
$$

Moreover, we denote $\chi_{\geq a}=1-\chi_{\leq a}$ and $\chi_{a \leq \leq b}=\chi_{\leq b}-\chi_{\leq a}$. We denote $\chi_{a}=\chi_{\leq 2 a}-\chi_{\leq a}$ for short.

Also, we need some Fourier operators. First, we recall the Fourier transform and its inverse formula. We denote the Fourier transform by $\hat{f}$ or $\mathscr{F} f$ as

$$
(\mathscr{F} f(\xi) \text { or }) \hat{f}(\xi)=\int_{\mathbb{R}^{d}} e^{-2 \pi i x \cdot \xi} f(x) d x
$$

and its inverse transform by $\check{f}$ or $\mathscr{F}^{-1} f$ as

$$
\left(\mathscr{F}^{-1} f(x) \text { or }\right) \check{f}(x)=\int_{\mathbb{R}^{d}} e^{2 \pi i x \cdot \xi} f(\xi) d \xi .
$$


For each number $N>0$, we define the Fourier multipliers $P_{\leq N}, P_{>N}, P_{N}$ as

$$
\begin{aligned}
\widehat{P_{\leq N} f}(\xi) & :=\chi_{\leq N}(\xi) \hat{f}(\xi), \\
\widehat{P_{>N} f}(\xi) & :=\chi_{>N}(\xi) \hat{f}(\xi), \\
\widehat{P_{N} f}(\xi) & :=\chi_{N}(\xi) \hat{f}(\xi),
\end{aligned}
$$

and similarly $P_{<N}$ and $P_{\geq N}$. We will usually use these multipliers when $N$ are dyadic numbers (that is, of the form $2^{k}$ for some integer $k$ ).

Moreover, we also need the notations

$$
\nabla_{\xi}=\left\{\partial_{\xi_{1}}, \cdots, \partial_{\xi_{d}}\right\} ; \quad \partial_{\xi}^{l}=\partial_{\xi_{1}}^{l^{1}} \cdots \partial_{\xi_{d}}^{l^{d}}, \text { for any } l=\left\{l^{1}, \cdots, l^{d}\right\} \in \mathbb{R}^{d} .
$$

2.2. Some basic lemmas. First, we need the following radial Sobolev embedding, see 62 for example.

Lemma 2.1. Let $\alpha, q, p, s$ be the parameters which satisfy

$$
\alpha>-\frac{d}{q} ; \quad \frac{1}{q} \leq \frac{1}{p} \leq \frac{1}{q}+s ; \quad 1 \leq p, q \leq \infty ; \quad 0<s<d
$$

with

$$
\alpha+s=d\left(\frac{1}{p}-\frac{1}{q}\right)
$$

Moreover, at most one of the equalities hold:

$$
p=1, \quad p=\infty, \quad q=1, \quad q=\infty, \quad \frac{1}{p}=\frac{1}{q}+s .
$$

Then

$$
\left\||x|^{\alpha} u\right\|_{L^{q\left(\mathbb{R}^{d}\right)}} \lesssim\left\||\nabla|^{s} u\right\|_{L^{p}\left(\mathbb{R}^{d}\right)} .
$$

The second is the following fractional Leibniz rule, see [34, 4, 46] and the references therein.

Lemma 2.2. Let $0<s<1, \frac{1}{2}<p \leq \infty$, and $1<p_{1}, p_{2}, p_{3}, p_{4} \leq \infty$ with $\frac{1}{p}=\frac{1}{p_{1}}+\frac{1}{p_{2}}$, $\frac{1}{p}=\frac{1}{p_{3}}+\frac{1}{p_{4}}$, and let $f, g \in \mathcal{S}\left(\mathbb{R}^{d}\right)$, then

$$
\left\||\nabla|^{s}(f g)\right\|_{L^{p}} \lesssim\left\||\nabla|^{s} f\right\|_{L^{p_{1}}}\|g\|_{L^{p_{2}}}+\left\||\nabla|^{s} g\right\|_{L^{p_{3}}}\|f\|_{L^{p_{4}}}
$$

A simple consequence is the following elementary inequality.

Lemma 2.3. For any $a>0,1 \leq p \leq \infty, 0 \leq \gamma<\frac{d}{p}$, and $|\nabla|^{\gamma} g \in L^{p}\left(\mathbb{R}^{d}\right)$,

$$
\left\||\nabla|^{\gamma}\left(\chi_{\leq a} g\right)\right\|_{L^{p}\left(\mathbb{R}^{d}\right)} \lesssim\left\||\nabla|^{\gamma} g\right\|_{L^{p}\left(\mathbb{R}^{d}\right)} .
$$

Here the implicit constant is independent on a. The same estimate holds for $\chi_{\geq a} g$.

Proof. The case $\gamma=0$ is trivial. Further, we may assume that $0<\gamma<1$. Otherwise, we can use the standard Leibniz rule and the Hölder inequality to reduce the derivatives.

From Lemma 2.2, the Hölder and Sobolev inequalities, we have

$$
\begin{aligned}
\left\||\nabla|^{\gamma}\left(\chi_{\leq a} g\right)\right\|_{L^{p}\left(\mathbb{R}^{d}\right)} & \lesssim\left\|\left.\nabla\right|^{\gamma} \chi_{\leq a}\right\|_{L^{\frac{d}{\gamma}\left(\mathbb{R}^{d}\right)}}\|g\|_{L^{\frac{d p}{d-p \gamma}\left(\mathbb{R}^{d}\right)}}+\left\|\chi_{\leq a}\right\|_{L^{\infty}\left(\mathbb{R}^{d}\right)}\left\||\nabla|^{\gamma} g\right\|_{L^{p}\left(\mathbb{R}^{d}\right)} \\
& \lesssim\left(\left\||\nabla|^{\gamma} \chi_{\leq a}\right\|_{L^{\frac{d}{\gamma}\left(\mathbb{R}^{d}\right)}}+\left\|\chi_{\leq a}\right\|_{L^{\infty}\left(\mathbb{R}^{d}\right)}\right)\left\||\nabla|^{\gamma} g\right\|_{L^{p}\left(\mathbb{R}^{d}\right)} .
\end{aligned}
$$


Note that $\left\|\chi_{\leq a}\right\|_{L^{\infty}\left(\mathbb{R}^{d}\right)} \lesssim 1$ and

$$
\left\||\nabla|^{\gamma} \chi_{\leq a}\right\|_{L^{\frac{d}{\gamma}\left(\mathbb{R}^{d}\right)}}=a^{-\gamma}\left\||\nabla|^{\gamma} \chi_{\leq 1}\left(\frac{\dot{-}}{a}\right)\right\|_{L^{\frac{d}{\gamma}}\left(\mathbb{R}^{d}\right)}=\left\||\nabla|^{\gamma} \chi_{\leq 1}\right\|_{L^{\frac{d}{\gamma}\left(\mathbb{R}^{d}\right)}} \lesssim 1
$$

Hence we obtain (2.1).

Note that

then by (2.1), we have

$$
\chi_{\geq a} g=1-\chi_{\leq a} g,
$$

$$
\left\|\chi_{\geq a} g\right\|_{\dot{H}^{\gamma}\left(\mathbb{R}^{d}\right)} \lesssim\|g\|_{\dot{H}^{\gamma\left(\mathbb{R}^{d}\right)}}+\left\|\chi_{\leq a} g\right\|_{\dot{H}^{\gamma\left(\mathbb{R}^{d}\right)}} \lesssim\|g\|_{\dot{H}^{\gamma}\left(\mathbb{R}^{d}\right)} .
$$

Hence, the same estimate holds for $\chi_{\leq a} g$. Thus we finish the proof of the lemma.

Note that the condition $\gamma<\frac{d}{p}$ in Lemma 2.3 can be removed if $a \gtrsim 1$. We also need the following Littlewood-Paley inequality, see for example [64].

Lemma 2.4. Let $p \in(1,+\infty)$ and $f \in L^{p}\left(\mathbb{R}^{d}\right)$. Then

$$
\|f\|_{L^{p}\left(\mathbb{R}^{d}\right)} \sim\left\|P_{\leq 1} f\right\|_{L^{p}\left(\mathbb{R}^{d}\right)}+\left\|\left(\sum_{j=1}^{\infty}\left|P_{j} f\right|^{2}\right)^{\frac{1}{2}}\right\|_{L^{p}\left(\mathbb{R}^{d}\right)} .
$$

Moreover, we need the following mismatch result, which is helpful in commuting the spatial and the frequency cutoffs.

Lemma 2.5 (Mismatch estimates, see [47]). Let $\phi_{1}$ and $\phi_{2}$ be smooth functions obeying

$$
\left|\phi_{j}\right| \leq 1 \quad \text { and } \operatorname{dist}\left(\operatorname{supp} \phi_{1}, \operatorname{supp} \phi_{2}\right) \geq A \text {, }
$$

for some large constant $A$. Then for $\sigma>0, M \leq 1$ and $1 \leq r \leq q \leq \infty$,

$$
\begin{gathered}
\left\|\phi_{1}|\nabla|^{\sigma} P_{\leq M}\left(\phi_{2} f\right)\right\|_{L_{x}^{q}\left(\mathbb{R}^{d}\right)}+\left\|\phi_{1} \nabla|\nabla|^{\sigma-1} P_{\leq M}\left(\phi_{2} f\right)\right\|_{L_{x}^{q}\left(\mathbb{R}^{d}\right)} \lesssim A^{-\sigma-\frac{d}{r}+\frac{d}{q}}\left\|\phi_{2} f\right\|_{L_{x}^{r}\left(\mathbb{R}^{d}\right)} ; \\
\left\|\phi_{1} \nabla P_{\leq M}\left(\phi_{2} f\right)\right\|_{L_{x}^{q}\left(\mathbb{R}^{d}\right)} \lesssim m M^{1-m} A^{-m}\|f\|_{L_{x}^{q}\left(\mathbb{R}^{d}\right)}, \text { for any } m \geq 0 .
\end{gathered}
$$

Furthermore, we need the following elementary formulas. The first one is

Lemma 2.6. Let the scale function $g \in \mathcal{S}\left(\mathbb{R}^{d}\right)$, and the phase function $\phi \in\left(\mathcal{S}\left(\mathbb{R}^{d}\right)\right)$ with

$$
\inf _{y \in \mathbb{R}^{d}}|\nabla \phi(y)|>0,
$$

then for any integer $N$,

$$
\int_{\mathbb{R}^{d}} e^{i \phi(y)} g(y) d y=\int_{\mathbb{R}^{d}} e^{i \phi(y)} \nabla_{y} \cdot\left(\frac{\nabla_{y} \phi}{i\left|\nabla_{y} \phi\right|^{2}} \nabla_{y}\right)^{N-1} \cdot\left(\frac{\nabla_{y} \phi}{i\left|\nabla_{y} \phi\right|^{2}} g(y)\right) d y .
$$

Proof. Note that

Then we write

$$
e^{i \phi}=\nabla_{y} e^{i \phi} \cdot \frac{\nabla_{y} \phi}{i\left|\nabla_{y} \phi\right|^{2}} .
$$

By integration-by-parts, we get

$$
\int_{\mathbb{R}^{d}} e^{i \phi(y)} g(y) d y=\int_{\mathbb{R}^{d}} \nabla_{y} e^{i \phi} \cdot \frac{\nabla_{y} \phi}{i\left|\nabla_{y} \phi\right|^{2}} g(y) d y .
$$

$$
\int_{\mathbb{R}^{d}} e^{i \phi(y)} g(y) d y=\int_{\mathbb{R}^{d}} e^{i \phi} \nabla_{y} \cdot\left(\frac{\nabla_{y} \phi}{i\left|\nabla_{y} \phi\right|^{2}} g(y)\right) d y .
$$


This obtains the case of $N=1$. Denote that

then we have

$$
\Lambda_{N}(g)=\nabla_{y} \cdot\left(\frac{\nabla_{y} \phi}{i\left|\nabla_{y} \phi\right|^{2}} \nabla_{y}\right)^{N-1} \cdot\left(\frac{\nabla_{y} \phi}{i\left|\nabla_{y} \phi\right|^{2}} g(y)\right),
$$

$$
\Lambda_{N+1}(g)=\nabla_{y} \cdot\left(\frac{\nabla_{y} \phi}{i\left|\nabla_{y} \phi\right|^{2}} \Lambda_{N}(g)\right) .
$$

The identity is then followed from the induction.

The second one is

Lemma 2.7. Let the vector function $f \in\left(\mathcal{S}\left(\mathbb{R}^{d}\right)\right)^{d}$ and the scale function $g \in \mathcal{S}\left(\mathbb{R}^{d}\right)$, then for any integer $N$,

$$
\nabla_{\xi} \cdot\left(f \nabla_{\xi}\right)^{N-1} \cdot(f g)=\sum_{\substack{l_{1}, \cdots, l_{N} \in \mathbb{R}^{d}, l^{\prime} \in \mathbb{R}^{d} ; \\\left|l_{j}\right| \leq j ;\left|l_{1}\right|+\cdots+\left|l_{N}\right|+\left|l^{\prime}\right|=N}} C_{l_{1}, \cdots, l_{N}, l^{\prime}} \partial_{\xi}^{l_{1}} f \cdots \partial_{\xi}^{l_{N}} f \partial_{\xi}^{l^{\prime}} g .
$$

Proof. When $N=1$, it is directly followed from the Leibniz rule. Denote that

$$
\Lambda_{N}(f, g)=\nabla_{\xi} \cdot\left(f \nabla_{\xi}\right)^{N-1} \cdot(f g),
$$

then we have

$$
\Lambda_{N}(f, g)=\nabla_{\xi} \cdot\left(f \Lambda_{N-1}(f, g)\right) .
$$

The identity is then followed from the induction.

2.3. Linear Schrödinger operator. Let the operator $S(t)=e^{i t \Delta}$ be the linear Schrödinger flow, that is,

$$
\left(i \partial_{t}+\Delta\right) S(t) \equiv 0
$$

The following are some fundamental properties of the operator $e^{i t \Delta}$. The first is the explicit formula, see for example Cazenave [9].

Lemma 2.8. For all $\phi \in \mathcal{S}\left(\mathbb{R}^{d}\right), t \neq 0$,

$$
S(t) \phi(x)=\frac{1}{(4 \pi i t)^{\frac{d}{2}}} \int_{\mathbb{R}^{d}} e^{\frac{i|x-y|^{2}}{4 t}} \phi(y) d y .
$$

Moreover, for any $r \geq 2$,

$$
\|S(t) \phi\|_{L_{x}^{r}\left(\mathbb{R}^{d}\right)} \lesssim|t|^{-d\left(\frac{1}{2}-\frac{1}{r}\right)}\|\phi\|_{L^{r^{\prime}\left(\mathbb{R}^{d}\right)}}
$$

The following is the standard Strichartz estimates, see for example [29].

Lemma 2.9. Let $I$ be a compact time interval and let $u: I \times \mathbb{R}^{d} \rightarrow \mathbb{R}$ be a solution to the inhomogeneous Schrödinger equation

$$
i u_{t}-\Delta u+F=0 .
$$

Then for any $t_{0} \in I$, any pairs $\left(q_{j}, r_{j}\right), j=1,2$ satisfying

$$
q_{j} \geq 2, r_{j} \geq 2, \text { and } \frac{2}{q_{j}}+\frac{d}{r_{j}}=\frac{d}{2},
$$

the following estimates hold,

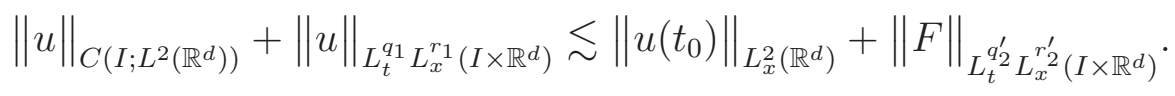


We also need the special Strichartz estimates for radial data, which was firstly proved by Shao [60], and then developed in [11, 28].

Lemma 2.10 (Radial Strichartz estimates). Let $g \in L^{2}\left(\mathbb{R}^{d}\right)$ be a radial function, then for any triple $(q, r, \gamma)$ satisfying

$$
\gamma \in \mathbb{R}, q \geq 2, r>2, \frac{2}{q}+\frac{2 d-1}{r}<\frac{2 d-1}{2}, \text { and } \frac{2}{q}+\frac{d}{r}=\frac{d}{2}+\gamma,
$$

we have that

$$
\left\||\nabla|^{\gamma} e^{i t \Delta} g\right\|_{L_{t}^{q} L_{x}^{r}\left(\mathbb{R} \times \mathbb{R}^{d}\right)} \lesssim\|g\|_{L^{2}\left(\mathbb{R}^{d}\right)} .
$$

Followed by the standard $\mathrm{TT}^{*}$-method, a direct consequence of the lemma above is

Corollary 2.11 (Inhomogeneous radial Strichartz estimates). Let $F \in L_{t}^{\tilde{q}^{\prime}} L_{x}^{\tilde{r}^{\prime}}\left(\mathbb{R}^{d+1}\right)$ be a radial function in $x$, then

$$
\left\|\int_{0}^{t} e^{i(t-s) \Delta}|\nabla|^{\gamma} F(s) d s\right\|_{L_{t}^{q} L_{x}^{r}\left(\mathbb{R}^{1+d}\right)} \lesssim\left\||\nabla|^{-\tilde{\gamma}} F\right\|_{L_{t}^{\tilde{q}^{\prime}} L_{x}^{\tilde{r}^{\prime}}\left(\mathbb{R}^{1+d}\right)},
$$

where the triples $(q, r, \gamma),(\tilde{q}, \tilde{r}, \tilde{\gamma})$ satisfy (2.5)

The following is a remark regarding Lemma 2.10 and Corollary 2.11

Remark 2.12. One may ask about the optimal smoothing effect one can gain from the radial Strichartz estimates, corresponding to the supremum of $\gamma$ as above. In fact, from Lemma 2.10, we find that

$$
\gamma<\frac{2}{q} \cdot \frac{d-1}{2 d-1}
$$

An equivalent inequality is

$$
\gamma<(d-1)\left(\frac{1}{2}-\frac{1}{r}\right)
$$

Hence, $\frac{2}{q} \cdot \frac{d-1}{2 d-1}-$ is the most derivatives we can gain from the radial Strichartz estimates when we fix $q$; while $(d-1)\left(\frac{1}{2}-\frac{1}{r}\right)$ - is the most derivatives we can gain from the radial Strichartz estimates when we fix $r$. In particular, when $q=2$, we denote the triples

$$
\left(q_{0}, r_{0}, \gamma_{0}\right):=\left(2, \frac{2(2 d-1)}{2 d-3}+, \frac{d-1}{2 d-1}-\right) .
$$

Therefore, $\gamma_{0}=\frac{d-1}{2 d-1}-$ is the most derivatives we can gain from the radial Strichartz estimates. While we can gain double derivatives from the inhomogeneous radial Strichartz estimates by setting $\tilde{\gamma}=\gamma$ in Corollary 2.11.

Further, we note that from Corollary 2.11, for any $h \in L_{t}^{2} L_{x}^{r_{0}^{\prime}}\left(\mathbb{R} \times \mathbb{R}^{d}\right)$,

$$
\left\|\int_{0}^{t} e^{i(t-s) \Delta}|\nabla|^{\gamma_{0}} h(s) d s\right\|_{L_{t}^{2} L_{x}^{\frac{2 d}{d-2}}\left(\mathbb{R} \times \mathbb{R}^{d}\right)} \lesssim\|h\|_{L_{t}^{2} L_{x}^{r_{0}^{\prime}}\left(\mathbb{R} \times \mathbb{R}^{d}\right)} .
$$

Further, by Sobolev's inequality, it infers that

$$
\left\|\int_{0}^{t} e^{i(t-s) \Delta} h(s) d s\right\|_{L_{t}^{2} L_{x}^{\rho_{0}\left(\mathbb{R} \times \mathbb{R}^{d}\right)}} \lesssim\|h\|_{L_{t}^{2} L_{x}^{r_{0}^{\prime}\left(\mathbb{R} \times \mathbb{R}^{d}\right)}},
$$

where $\rho_{0}$ satisfies that

$$
\frac{1}{\rho_{0}}=\frac{d-2}{2 d}-\frac{\gamma_{0}}{d}=\frac{1}{2}-\frac{3 d-2}{d(2 d-1)}+
$$


Now we need the following specific truncated inhomogeneous radial Strichartz estimates.

Lemma 2.13. Let $T \geq 2, r \geq \rho_{0}$, and let the triples $(\tilde{q}, \tilde{r}, \tilde{\gamma})$ satisfy that for some $\theta \in[0,1]$,

$$
\frac{1}{\tilde{q}} \leq(1-\theta)+\frac{1}{2} \theta ; \quad \frac{1}{\tilde{r}}=\frac{1-\theta}{r^{\prime}}+\frac{\theta}{r_{0}^{\prime}} ; \quad \tilde{\gamma}=\theta\left(\frac{d-2}{2}-\gamma_{0}-\frac{d}{r}\right) .
$$

Suppose that $F$ is a radial function in $x$ such that $|\nabla|^{\tilde{\gamma}} F \in L_{t}^{\tilde{q}} L_{x}^{\tilde{r}}\left([0,2] \times \mathbb{R}^{d}\right)$, then

$$
\left\|\int_{0}^{t} e^{i(t-s) \Delta} \chi_{\leq 1}(s) F(s) d s\right\|_{L_{t}^{1} L_{x}^{r}\left([T, 2 T] \times \mathbb{R}^{d}\right)} \lesssim T^{\left[-d\left(\frac{1}{2}-\frac{1}{r}\right)+1\right](1-\theta)+\frac{1}{2} \theta}\||\nabla| \tilde{\gamma} F\|_{L_{t}^{\tilde{q}} L_{x}^{\tilde{r}}\left([0,2] \times \mathbb{R}^{d}\right)} .
$$

Proof. By Lemma 2.8,

$$
\left\|\int_{0}^{t} e^{i(t-s) \Delta} \chi_{\leq 1}(s) F(s) d s\right\|_{L_{x}^{r}\left(\mathbb{R}^{d}\right)} \lesssim \int_{0}^{t}|t-s|^{-d\left(\frac{1}{2}-\frac{1}{r}\right)} \chi_{\leq 1}(s)\|F(s)\|_{L_{x}^{r^{\prime}\left(\mathbb{R}^{d}\right)}} d s .
$$

Since $t-s \sim t$ when $t \geq 2, s \leq \frac{11}{10}$, we further have that

$$
\left\|\int_{0}^{t} e^{i(t-s) \Delta} \chi_{\leq 1}(s) F(s) d s\right\|_{L_{x}^{r}\left(\mathbb{R}^{d}\right)} \lesssim|t|^{-d\left(\frac{1}{2}-\frac{1}{r}\right)}\|F\|_{L_{t}^{1} L_{x}^{r^{\prime}\left([0,2] \times \mathbb{R}^{d}\right)}} .
$$

This gives that

$$
\left\|\int_{0}^{t} e^{i(t-s) \Delta} \chi_{\leq 1}(s) F(s) d s\right\|_{L_{t}^{1} L_{x}^{r}\left([T, 2 T] \times \mathbb{R}^{d}\right)} \lesssim T^{-d\left(\frac{1}{2}-\frac{1}{r}\right)+1}\|F\|_{L_{t}^{1} L_{x}^{r^{\prime}}\left([0,2] \times \mathbb{R}^{d}\right)} .
$$

On the other hand, applying the Sobolev embedding and the inequality (2.6), we obtain that for any $r \geq \rho_{0}$,

$$
\begin{aligned}
\left\|\int_{0}^{t} e^{i(t-s) \Delta} \chi_{\leq 1}(s) F(s) d s\right\|_{L_{t}^{2} L_{x}^{r}\left(\mathbb{R} \times \mathbb{R}^{d}\right)} & \lesssim\left\|\int_{0}^{t} e^{i(t-s) \Delta} \chi_{\leq 1}(s)|\nabla|^{d\left(\frac{1}{\rho_{0}}-\frac{1}{r}\right)} F(s) d s\right\|_{L_{t}^{2} L_{x}^{\rho_{0}}\left(\mathbb{R} \times \mathbb{R}^{d}\right)} \\
& \lesssim\left\|\chi_{\leq 1}(t)|\nabla|^{d\left(\frac{1}{\rho_{0}}-\frac{1}{r}\right)} F(t)\right\|_{L_{t}^{2} L_{x}^{r_{0}^{\prime}}\left(\mathbb{R} \times \mathbb{R}^{d}\right)}
\end{aligned}
$$

Therefore, by the time support, we get that

$$
\left\|\int_{0}^{t} e^{i(t-s) \Delta} \chi_{\leq 1}(s) F(s) d s\right\|_{L_{t}^{1} L_{x}^{r}\left([T, 2 T] \times \mathbb{R}^{d}\right)} \lesssim T^{\frac{1}{2}}\left\||\nabla|^{d\left(\frac{1}{\rho_{0}}-\frac{1}{r}\right)} F\right\|_{L_{t}^{2} L_{x}^{r_{0}^{\prime}\left([0,2] \times \mathbb{R}^{d}\right)}} .
$$

Then by interpolation between (2.7), (2.8) and Hölder's inequality in time, we obtain the desired estimates.

Remark 2.14. Note that if

$$
\left(-d\left(\frac{1}{2}-\frac{1}{r}\right)+1\right)(1-\theta)+\frac{1}{2} \theta<0,
$$

then by dyadic decomposition in time, we have that

$$
\left\|\int_{0}^{t} e^{i(t-s) \Delta} \chi_{\leq 1}(s) F(s) d s\right\|_{L_{t}^{1} L_{x}^{r}\left([2,+\infty) \times \mathbb{R}^{d}\right)} \lesssim\left\||\nabla|^{\tilde{\gamma}} F\right\|_{L_{t}^{\tilde{q}} L_{x}^{\tilde{r}}\left([0,2] \times \mathbb{R}^{d}\right)} .
$$




\section{LiNEAR FLOW ESTIMATES ON LOCALIZED FUNCTIONS}

As the first part, we begin with the linear flow estimates. In this section, we give the following estimates.

Proposition 3.1. Let $2 \leq r<\infty$, then for any $t:|t| \geq \frac{100}{N}$, and any $s$ satisfying

$$
0 \leq s<(d-2)\left(\frac{1}{2}-\frac{1}{r}\right)+s_{c}
$$

the following estimate holds,

$$
\left\||\nabla|^{s}\left(e^{i t \Delta}\left(\chi_{\leq 10} P_{\geq N} g\right)\right)\right\|_{L_{x}^{r}\left(\mathbb{R}^{d}\right)} \lesssim N^{-(d-2)\left(\frac{1}{2}-\frac{1}{r}\right)+s-s_{c}}|t|^{-(d-1)\left(\frac{1}{2}-\frac{1}{r}\right)}\left\|P_{\geq N} g\right\|_{\dot{H}^{s_{c}}\left(\mathbb{R}^{d}\right)} .
$$

Moreover, let $d \geq 3,(q, r, \gamma)$ be the triple satisfying (2.5) and

$$
2 \leq q<\infty, \quad \frac{1}{q}<(d-1)\left(\frac{1}{2}-\frac{1}{r}\right) .
$$

Then there exist $s_{*}=s_{*}(q)<0, \eta_{*}=\eta_{*}(q, r, \gamma)>0$, such that for any $\alpha \geq 1, \beta>0$ with $\alpha \beta \leq \eta_{*}$, and any $s_{c} \in\left[s_{*}, 0\right)$, the following estimate holds,

$$
\left\|\left\langle t^{\alpha}|\nabla|\right\rangle^{\beta}|\nabla|^{s_{c}+\gamma}\left(e^{i t \Delta} \chi_{\leq 10}\left(P_{\geq N} g\right)\right)\right\|_{L_{t}^{q} L_{x}^{r}\left(\mathbb{R} \times \mathbb{R}^{d}\right)} \lesssim\left\|P_{\geq N} g\right\|_{H^{s_{c}}\left(\mathbb{R}^{d}\right)} .
$$

Remark 3.2. From the proof of Proposition 3.1 below, it also follows that $s_{*}(q)$ can be chosen to be a increasing function with $s_{*}(+\infty)=0$. What we use below is $s_{*}(2)$.

The proof of the proposition is based on the following two lemmas and corollaries. First of all, we show the estimate in the local domain.

Lemma 3.3. Let $M \geq 1, r \geq 2$ and $s \geq 0$, then for any $t:|t| \geq \frac{100}{M}$, any $K \in \mathbb{Z}^{+}$,

$$
\left\||\nabla|^{s}\left(\chi_{\leq \frac{1}{10} M|t|} e^{i t \Delta}\left(\chi_{\leq 10} P_{M} g\right)\right)\right\|_{L_{x}^{r}\left(\mathbb{R}^{d}\right)} \lesssim_{s, K}|t|^{-d\left(\frac{1}{2}-\frac{1}{r}\right)} M^{-K}\left\|P_{M} g\right\|_{H^{s_{c}}\left(\mathbb{R}^{d}\right)} .
$$

Proof. First, we show that for any $M \geq 1, t:|t| \geq \frac{100}{M}, s \in \mathbb{Z}^{+} \cup\{0\}$ and $K \in \mathbb{Z}^{+}$,

$$
\left.\left.|| \nabla\right|^{s}\left(\chi_{\leq \frac{1}{10} M|t|} e^{i t \Delta}\left(\chi_{\leq 10} P_{M} g\right)\right)\left|\lesssim_{K}\right| t\right|^{-\frac{d}{2}} M^{-K}\left\|P_{M} g\right\|_{\dot{H}^{s^{c}}\left(\mathbb{R}^{d}\right)} .
$$

To show this, we use the formulas in Lemma 2.8 and the inverse Fourier transform to obtain

$$
\begin{aligned}
e^{i t \Delta}\left(\chi_{\leq 10} P_{M} g\right)(x) & =\frac{1}{(4 \pi i t)^{\frac{d}{2}}} \int_{\mathbb{R}^{d}} e^{\frac{i|x-y|^{2}}{4 t}} \chi_{\leq 10}(y) P_{M} g(y) d y \\
& =\frac{1}{(4 \pi i t)^{\frac{d}{2}}} \int_{\mathbb{R}^{d}} \int_{\mathbb{R}^{d}} e^{\frac{i|x-y|^{2}}{4 t}+2 \pi i y \cdot \xi} \chi_{\leq 10}(y) \chi_{M}(\xi) \hat{g}(\xi) d y d \xi
\end{aligned}
$$

Fix $x, \xi$, and define the phase as

$$
\phi(y)=-\frac{x \cdot y}{2 t}+\frac{|y|^{2}}{4 t}+y \cdot \xi
$$

then from (3.6),

$$
e^{i t \Delta}\left(\chi_{\leq 10} P_{M} g\right)(x)=\frac{e^{\frac{i|x|^{2}}{4 t}}}{(4 \pi i t)^{\frac{d}{2}}} \int_{\mathbb{R}^{d}} \int_{\mathbb{R}^{d}} e^{i \phi(y)} \chi_{\leq 10}(y) \chi_{M}(\xi) \hat{g}(\xi) d y d \xi .
$$


Moreover, we have

$$
\nabla_{y} \phi(y)=\frac{y-x}{2 t}+\xi
$$

and

$$
\partial_{y_{j} y_{k}} \phi(y)=\frac{\delta_{j k}}{2 t}, \quad \partial_{y_{j} y_{k} y_{h}} \phi(y)=0, \text { for any } j, k, h \in\{1, \cdots, d\} .
$$

Note that

$$
|t| M \geq 100,|\xi| \geq \frac{9}{10} M,|y| \leq 11, \text { and }|x| \leq \frac{11}{100} M|t|,
$$

from (3.8) we have

$$
|\nabla \phi(y)| \gtrsim|\xi|
$$

Then using the formula (2.4) to the right-hand side of (3.7), we obtain

$$
\begin{aligned}
\chi_{\leq \frac{1}{10} M|t|}(x) e^{i t \Delta}\left(\chi_{\leq 10} P_{M} g\right)(x)=\chi_{\leq \frac{1}{10} M|t|}(x) \frac{C_{K} e^{\frac{i|x|^{2}}{4 t}}}{t^{\frac{d}{2}}} \int_{\mathbb{R}^{d}} \int_{\mathbb{R}^{d}} e^{i \phi(y)} \\
\cdot \nabla_{y} \cdot\left(\frac{\nabla_{y} \phi}{i\left|\nabla_{y} \phi\right|^{2}} \nabla_{y}\right)^{K-1} \cdot\left(\frac{\nabla_{y} \phi}{i\left|\nabla_{y} \phi\right|^{2}} \chi_{\leq 10}(y)\right) d y \cdot \chi_{M}(\xi) \hat{g}(\xi) d \xi .
\end{aligned}
$$

We claim that

$$
\left|\nabla_{y} \cdot\left(\frac{\nabla_{y} \phi}{i\left|\nabla_{y} \phi\right|^{2}} \nabla_{y}\right)^{K-1} \cdot\left(\frac{\nabla_{y} \phi}{i\left|\nabla_{y} \phi\right|^{2}} \chi_{\leq 10}(y)\right)\right| \lesssim|\xi|^{-K} \chi_{\lesssim 1}(\cdot) .
$$

Indeed, from Lemma 2.7, we expand the left-hand side of (3.12) as

$$
\sum_{\substack{l_{1}, \cdots, l_{K} \in \mathbb{R}^{d}, l^{\prime} \in \mathbb{R}^{d} ; \\\left|l_{j}\right| \leq j ;\left|l_{1}\right|+\cdots+\left|l_{K}\right|+\left|l^{\prime}\right|=K}} C_{l_{1}, \cdots, l_{K}, l^{\prime}} \partial_{y}^{l_{1}}\left(\frac{\nabla_{y} \phi}{i\left|\nabla_{y} \phi\right|^{2}}\right) \cdots \partial_{y}^{l_{K}}\left(\frac{\nabla_{y} \phi}{i\left|\nabla_{y} \phi\right|^{2}}\right) \partial_{y}^{l^{\prime}}\left(\chi_{\leq 10}(\cdot)\right) .
$$

Note that from (3.9) and (3.10), we have that for any non-negative integer vectors $l, l^{\prime}$,

$$
\left|\partial_{y}^{l}\left(\frac{\nabla_{y} \phi}{i\left|\nabla_{y} \phi\right|^{2}}\right)\right| \lesssim \frac{1}{|\xi|} \frac{1}{|t \xi|^{|l|}}
$$

and

$$
\left|\partial_{y}^{l^{\prime}}\left(\chi_{\leq 10}(\cdot)\right)\right| \lesssim \chi_{\lesssim 1}(\cdot)
$$

Hence, using these two estimates, and noting that $|t \xi| \gtrsim 1$, we have that

$$
\begin{aligned}
\mid(\underline{3.13)} \mid & \lesssim \sum_{\substack{l_{1}, \ldots, l_{K} \in \mathbb{R}^{d}, l^{\prime} \in \mathbb{R}^{d} ; \\
\left|l_{j}\right| \leq j ;\left|l_{1}\right|+\cdots+\left|l_{K}\right|+\left|l^{\prime}\right|=K}} \frac{1}{|\xi|} \frac{1}{|t \xi|^{\left|l_{1}\right|}} \cdots \frac{1}{|\xi|} \frac{1}{|t \xi|^{\left|l_{K}\right|}} \cdot \chi_{\lesssim 1}(y) \\
& =\sum_{\substack{l_{1}, \cdots, l_{K} \in \mathbb{R}^{d}, l^{\prime} \in \mathbb{R}^{d} ; \\
\left|l_{j}\right| \leq j ;\left|l_{1}\right|+\cdots+\left|l_{K}\right|+\left|l^{\prime}\right|=K}}|\xi|^{-K}|t \xi|^{-\left(\left|l_{1}\right|+\cdots+\left|l_{K}\right|\right)} \cdot \chi_{\lesssim 1}(y) \\
& \lesssim|\xi|^{-K} \chi_{\lesssim 1}(y) .
\end{aligned}
$$

Therefore, we obtain (3.12). 
Inserting (3.12) into (3.11), we obtain

$$
\begin{aligned}
\left|\chi_{\leq \frac{1}{10} M|t|} e^{i t \Delta}\left(\chi_{\leq 10} P_{M} g\right)\right| & \lesssim|t|^{-\frac{d}{2}} \int_{\mathbb{R}^{d}} \int_{\mathbb{R}^{d}} \chi_{\lesssim 1}(y)|\xi|^{-K} \chi_{M}(\xi) \hat{g}(\xi) d y d \xi \\
& \lesssim|t|^{-\frac{d}{2}} M^{-K-s_{c}+d}\left\|P_{M} g\right\|_{\dot{H}^{s_{c}}\left(\mathbb{R}^{d}\right)} .
\end{aligned}
$$

Note that when the derivatives hit the cut-off functions $\chi_{\leq \frac{1}{10} M|t|}$ and $\chi_{\leq 10}$, the estimates on

$$
\chi_{\leq \frac{1}{10} M|t|} e^{i t \Delta}\left(\chi_{\leq 10} P_{M} g\right)
$$

become better, hence by choosing $K$ suitable large, we obtain that for any $s \in \mathbb{Z}^{+}$,

$$
\begin{aligned}
\left.|| \nabla\right|^{s}\left(\chi_{\leq \frac{1}{10} M|t|} e^{i t \Delta}\left(\chi_{\leq 10} P_{M} g\right)\right) \mid & \lesssim|t|^{-\frac{d}{2}} \int_{\mathbb{R}^{d}} \int_{\mathbb{R}^{d}} \chi_{\lesssim 1}(y)|\xi|^{-K} \chi_{M}(\xi) \hat{g}(\xi) d y d \xi \\
& \lesssim|t|^{-\frac{d}{2}} M^{-K-s_{c}+d}\left\|P_{M} g\right\|_{H^{s_{c}}\left(\mathbb{R}^{d}\right)} .
\end{aligned}
$$

Replacing $K$ by $K-s_{c}+d$, we obtain (3.5).

Further, using (3.5), Lemma 2.5, Hölder's inequality and interpolation when $s$ is not an integer, we obtain (3.4).

Then a direct consequence of the previous lemma is the following corollary.

Corollary 3.4. Let $(q, r, \gamma)$ be the triple satisfying the same conditions as in Proposition 3.1, and let $\alpha, \beta$ be the constants satisfying

$$
\alpha \geq 1, \quad \beta>0, \quad d\left(\frac{1}{2}-\frac{1}{r}\right)>\max \left\{\alpha \beta,-\alpha\left(s_{c}+\gamma\right)\right\}+\frac{1}{q} .
$$

Then there exists $s_{*, 1}=s_{*, 1}(q)<0$ such that for any $s_{c} \in\left[s_{*, 1}, 0\right)$, the following estimate holds,

$$
\left\|\left\langle t^{\alpha}|\nabla|\right\rangle^{\beta}|\nabla|^{s_{c}+\gamma}\left(\chi_{\leq \frac{1}{10} M|t|} e^{i t \Delta}\left(\chi_{\leq 10} P_{M} g\right)\right)\right\|_{L_{t}^{q} L_{x}^{r}\left(\mathbb{R} \times \mathbb{R}^{d}\right)} \lesssim\left\|P_{M} g\right\|_{\dot{H}^{s_{c}}\left(\mathbb{R}^{d}\right)} .
$$

Proof. We write

$$
\begin{aligned}
& \left\|\left\langle t^{\alpha}|\nabla|\right\rangle^{\beta}|\nabla|^{s_{c}+\gamma}\left(\chi_{\leq \frac{1}{10} M|t|} e^{i t \Delta}\left(\chi_{\leq 10} P_{M} g\right)\right)\right\|_{L_{t}^{q} L_{x}^{r}\left(\mathbb{R} \times \mathbb{R}^{d}\right)} \\
& \lesssim\left\|\left.\left\langle t^{\alpha}|\nabla|\right\rangle^{\beta}|\nabla|^{s_{c}+\gamma} P_{\leq|t|}\right|^{-\alpha}\left(\chi_{\leq \frac{1}{10} M|t|} e^{i t \Delta}\left(\chi_{\leq 10} P_{M} g\right)\right)\right\|_{L_{t}^{q} L_{x}^{r}\left(\mathbb{R} \times \mathbb{R}^{d}\right)} \\
& \quad+\left\|\left\langle t^{\alpha}|\nabla|\right\rangle^{\beta}|\nabla|^{s_{c}+\gamma} P_{\geq|t|-\alpha}\left(\chi_{\leq \frac{1}{10} M|t|} e^{i t \Delta}\left(\chi_{\leq 10} P_{M} g\right)\right)\right\|_{L_{t}^{q} L_{x}^{r}\left(\mathbb{R} \times \mathbb{R}^{d}\right)}
\end{aligned}
$$

For the first term (3.16a), we may use the Sobolev inequality to replace $(\gamma, r)$ by $(\gamma(q), r(q))$ which is defined by (see Remark 2.12)

$$
\gamma(q)=\frac{2}{q} \cdot \frac{d-1}{2 d-1}-, \quad \frac{1}{r(q)}=\frac{1}{2}-\frac{2}{q} \cdot \frac{1}{2 d-1}-.
$$

Then we set $s_{*} \geq-\gamma(q)$ such that $s_{c}+\gamma(q) \geq 0$. To simplify the notation, we still denote $(\gamma(q), r(q))$ by $(\gamma, r)$. Then

$$
\text { (3.16a) } \lesssim\left\||\nabla|^{s_{c}+\gamma}\left(\chi_{\leq \frac{1}{10} M|t|} e^{i t \Delta}\left(\chi_{\leq 10} P_{M} g\right)\right)\right\|_{L_{t}^{q} L_{x}^{r}\left(\mathbb{R} \times \mathbb{R}^{d}\right)} .
$$

Noting that $\gamma(q)<\frac{d}{r(q)}$, then using Lemma 2.3 and Lemma 2.10, it is controlled by $\left\|P_{M} g\right\|_{\dot{H}^{s_{c}}\left(\mathbb{R}^{d}\right)}$. 
For the second term (3.16b), noting that $\chi_{\leq R}=P_{\leq \frac{20}{R}} \chi_{\leq R}$, we have that

$$
\begin{aligned}
(3.16 \mathrm{~b}) & \lesssim\left\||t|^{\alpha \beta}|\nabla|^{s_{c}+\beta+\gamma} P_{\geq|t|^{-\alpha}}\left(\chi_{\leq \frac{1}{10} M|t|} e^{i t \Delta}\left(\chi_{\leq 10} P_{M} g\right)\right)\right\|_{L_{t}^{q} L_{x}^{r}\left(\mathbb{R} \times \mathbb{R}^{d}\right)} \\
& \lesssim\left\||t|^{\alpha \beta}|\nabla|^{s_{c}+\beta+\gamma} P_{\geq|t|^{-\alpha}} P_{\leq \frac{200}{M|t|}+2 M}\left(\chi_{\leq \frac{1}{10} M|t|} e^{i t \Delta}\left(\chi_{\leq 10} P_{M} g\right)\right)\right\|_{L_{t}^{q} L_{x}^{r}\left(\mathbb{R} \times \mathbb{R}^{d}\right)} .
\end{aligned}
$$

Since $\alpha \geq 1$, then due to the compatibility of frequency restriction

$$
P_{\geq|t|-\alpha} P_{\leq \frac{200}{M|t|}+2 M}
$$

we have that $|t| \gtrsim 1$. Hence, we get that

$$
(\underline{3.16 \mathrm{~b}}) \lesssim\left\||t|^{\alpha \beta}|\nabla|^{s_{c}+\beta+\gamma} P_{\geq|t|-\alpha} P_{\leq \frac{200}{M|t|}+2 M}\left(\chi_{\leq \frac{1}{10} M|t|} e^{i t \Delta}\left(\chi_{\leq 10} P_{M} g\right)\right)\right\|_{L_{t}^{q} L_{x}^{r}\left(\{|t| \gtrsim 1\} \times \mathbb{R}^{d}\right)}
$$

For (3.18), if $s_{c}+\beta+\gamma \geq 0$, then applying Lemma 3.3, we obtain that

$$
\left(\underline{3.18)} \lesssim\left\|t^{-d\left(\frac{1}{2}-\frac{1}{r}\right)+\alpha \beta}\right\|_{L_{t}^{q}(\{|t| \gtrsim 1\})} M^{-K}\left\|P_{M} g\right\|_{\dot{H}^{s_{c}\left(\mathbb{R}^{d}\right)}} \lesssim\left\|P_{M} g\right\|_{\dot{H}^{s_{c}\left(\mathbb{R}^{d}\right)}} .\right.
$$

Here we have used the condition of $d\left(\frac{1}{2}-\frac{1}{r}\right)>\alpha \beta+\frac{1}{q}$. If $s_{c}+\beta+\gamma<0$, then by Bernstein's inequality and Lemma 3.3, we get that

$$
\begin{aligned}
(3.18) & \lesssim\left\||t|^{-\left(s_{c}+\gamma\right) \alpha} P_{\geq|t|^{-\alpha}} P_{\leq \frac{200}{M|t|}+2 M}\left(\chi_{\leq \frac{1}{10} M|t|} e^{i t \Delta}\left(\chi_{\leq 10} P_{M} g\right)\right)\right\|_{L_{t}^{q} L_{x}^{r}\left(\{|t| \gtrsim 1\} \times \mathbb{R}^{d}\right)} \\
& \lesssim\left\||t|^{-\left(s_{c}+\gamma\right) \alpha}\left(\chi_{\leq \frac{1}{10} M|t|} e^{i t \Delta}\left(\chi_{\leq 10} P_{M} g\right)\right)\right\|_{L_{t}^{q} L_{x}^{r}\left(\{|t| \gtrsim 1\} \times \mathbb{R}^{d}\right)} \\
& \lesssim\left\|t^{-d\left(\frac{1}{2}-\frac{1}{r}\right)-\left(s_{c}+\gamma\right) \alpha}\right\|_{L_{t}^{q}(\{|t| \gtrsim 1\})} M^{-K}\left\|P_{M} g\right\|_{\dot{H}^{s_{c}\left(\mathbb{R}^{d}\right)}} \\
& \lesssim\left\|P_{M} g\right\|_{\dot{H}^{s^{c}\left(\mathbb{R}^{d}\right)}} .
\end{aligned}
$$

Here we have used the condition of $d\left(\frac{1}{2}-\frac{1}{r}\right)>-\alpha\left(s_{c}+\gamma\right)+\frac{1}{q}$. Hence, no matter in which cases, (3.18) is controlled by $\left\|P_{M} g\right\|_{\dot{H}^{s_{c}}\left(\mathbb{R}^{d}\right)}$. Thus we have that

$$
(3.16 \mathrm{~b}) \lesssim\left\|P_{M} g\right\|_{\dot{H}^{s}\left(\mathbb{R}^{d}\right)} .
$$

Together with the two estimates on (3.16), we get (3.15).

The second lemma shows the estimates of the linear flow in the domain far away from the origin.

Lemma 3.5. Let $M \geq 1,2 \leq r<\infty$ and $s \geq 0$, then for any $t:|t| \gtrsim \frac{1}{M}$,

$$
\left\||\nabla|^{s}\left(\chi_{\geq \frac{1}{10} M|t|} e^{i t \Delta}\left(\chi_{\leq 10} P_{M} g\right)\right)\right\|_{L_{x}^{r}\left(\mathbb{R}^{d}\right)} \lesssim M^{-(d-2)\left(\frac{1}{2}-\frac{1}{r}\right)+s}|t|^{-(d-1)\left(\frac{1}{2}-\frac{1}{r}\right)}\left\|P_{M} g\right\|_{L_{x}^{2}\left(\mathbb{R}^{d}\right)} .
$$

Proof. From the radial Sobolev embedding in Lemma 2.1, we have

$$
\begin{aligned}
\left\|\chi_{\geq \frac{1}{10} M|t|} e^{i t \Delta}\left(\chi_{\leq 10} P_{M} g\right)\right\|_{L_{x}^{r}\left(\mathbb{R}^{d}\right)} & \lesssim(M|t|)^{-(d-1)\left(\frac{1}{2}-\frac{1}{r}\right)}\left\||\nabla|^{\frac{1}{2}-\frac{1}{r}} e^{i t \Delta}\left(\chi_{\leq 10} P_{M} g\right)\right\|_{L_{x}^{2}\left(\mathbb{R}^{d}\right)} \\
& \lesssim(M|t|)^{-(d-1)\left(\frac{1}{2}-\frac{1}{r}\right)}\left\||\nabla|^{\frac{1}{2}-\frac{1}{r}}\left(\chi_{\leq 10} P_{M} g\right)\right\|_{L_{x}^{2}\left(\mathbb{R}^{d}\right)} .
\end{aligned}
$$


Using Lemma 2.3, we have

$$
\left\||\nabla|^{\frac{1}{2}-\frac{1}{r}}\left(\chi_{\leq 10} P_{M} g\right)\right\|_{L_{x}^{2}\left(\mathbb{R}^{d}\right)} \lesssim M^{\frac{1}{2}-\frac{1}{r}}\left\|P_{M} g\right\|_{L_{x}^{2}\left(\mathbb{R}^{d}\right)} .
$$

This last estimate combined with (3.21) yields

$$
\left\|\chi_{\geq \frac{1}{10} M|t|} e^{i t \Delta}\left(\chi_{\leq 10} P_{M} g\right)\right\|_{L_{x}^{r}\left(\mathbb{R}^{d}\right)} \lesssim M^{-(d-2)\left(\frac{1}{2}-\frac{1}{r}\right)}|t|^{-(d-1)\left(\frac{1}{2}-\frac{1}{r}\right)}\left\|P_{M} g\right\|_{L_{x}^{2}\left(\mathbb{R}^{d}\right)} .
$$

Similarly, we also obtain that for any $s \geq 0$, we have (3.20). Indeed, if the derivatives hit the cut-off functions $\chi_{\geq \frac{1}{10} M|t|}$ (since $M|t| \gtrsim 1$ ) and $\chi_{\leq 10}$, the analogous estimates become better. Hence by the same way as (3.22), we obtain the estimates above.

A consequence of Lemma 3.5 is

Corollary 3.6. Let $(q, r, \gamma)$ be the triple satisfying the same conditions as in Proposition 3.1, and let $\alpha, \beta$ be the constants satisfying $\alpha \geq 1, \beta>0$ and

$$
(d-1)\left(\frac{1}{2}-\frac{1}{r}\right)>\max \left\{\alpha \beta,-\alpha\left(s_{c}+\gamma\right)\right\}+\frac{1}{q},
$$

Then there exists $s_{*, 2}=s_{*, 2}(q)<0$ such that for any $s_{c} \in\left[s_{*, 2}, 0\right)$, the following estimate holds,

$$
\left\|\left\langle t^{\alpha}|\nabla|\right\rangle^{\beta}|\nabla|^{s_{c}+\gamma}\left(\chi_{\geq \frac{1}{10} M|t|} e^{i t \Delta}\left(\chi_{\leq 10} P_{M} g\right)\right)\right\|_{L_{t}^{q} L_{x}^{r}\left(\mathbb{R} \times \mathbb{R}^{d}\right)} \lesssim\left\|P_{M} g\right\|_{\dot{H}^{s_{c}}\left(\mathbb{R}^{d}\right)} .
$$

Proof. We decompose it into the following three terms.

$$
\begin{aligned}
& \left\|\left\langle t^{\alpha}|\nabla|\right\rangle^{\beta}|\nabla|^{s_{c}+\gamma}\left(\chi_{\geq \frac{1}{10} M|t|} e^{i t \Delta}\left(\chi_{\leq 10} P_{M} g\right)\right)\right\|_{L_{t}^{q} L_{x}^{r}\left(\mathbb{R} \times \mathbb{R}^{d}\right)} \\
& \lesssim\left\|\left\langle t^{\alpha}|\nabla|\right\rangle^{\beta}|\nabla|^{s_{c}+\gamma} P_{\leq|t|^{-\alpha}}\left(\chi_{\geq \frac{1}{10} M|t|} e^{i t \Delta}\left(\chi_{\leq 10} P_{M} g\right)\right)\right\|_{L_{t}^{q} L_{x}^{r}\left(\mathbb{R} \times \mathbb{R}^{d}\right)} \\
& \quad+\left\|\left\langle t^{\alpha}|\nabla|\right\rangle^{\beta}|\nabla|^{s_{c}+\gamma} P_{\geq|t|^{-\alpha}}\left(\chi_{\geq \frac{1}{10} M|t|} e^{i t \Delta}\left(\chi_{\leq 10} P_{M} g\right)\right)\right\|_{L_{t}^{q} L_{x}^{r}\left(\left\{|t| \leq(3 M)^{-\frac{1}{\alpha}}\right\} \times \mathbb{R}^{d}\right)} \\
& \quad+\left\|\left\langle t^{\alpha}|\nabla|\right\rangle^{\beta}|\nabla|^{s_{c}+\gamma} P_{\geq|t|^{-\alpha}}\left(\chi_{\geq \frac{1}{10} M|t|} e^{i t \Delta}\left(\chi_{\leq 10} P_{M} g\right)\right)\right\|_{L_{t}^{q} L_{x}^{r}\left(\left\{|t| \geq(3 M)^{-\frac{1}{\alpha}}\right\} \times \mathbb{R}^{d}\right)}
\end{aligned}
$$

For the term (3.25a), treating similarly as (3.16a), we use the Sobolev inequality to replace $(\gamma, r)$ by $(\gamma(q), r(q))$ which is defined in (3.17). Then we set $s_{*} \geq-\gamma(q)$ such that $s_{c}+\gamma(q) \geq$ 0 . Again, to simplify the notation, we still denote $(\gamma(q), r(q))$ by $(\gamma, r)$. Then using Lemma 2.3 and Lemma 2.10, we have

$$
\text { (3.25a) }) \lesssim\left\||\nabla|^{s_{c}+\gamma}\left(\chi_{\geq \frac{1}{10} M|t|} e^{i t \Delta}\left(\chi_{\leq 10} P_{M} g\right)\right)\right\|_{L_{t}^{q} L_{x}^{r}\left(\mathbb{R} \times \mathbb{R}^{d}\right)} \lesssim\left\|P_{M} g\right\|_{\dot{H}^{s_{c}\left(\mathbb{R}^{d}\right)}}
$$

For the term (3.25b), since

$$
|t|^{-\alpha} \geq 3 M
$$

we have

$$
\begin{aligned}
(\underline{3.25 \mathrm{~b}}) & \lesssim\left\||t|^{\alpha \beta}|\nabla|^{s_{c}+\beta+\gamma} P_{\geq|t|-\alpha}\left(\chi_{\geq \frac{1}{10} M|t|} e^{i t \Delta}\left(\chi_{\leq 10} P_{M} g\right)\right)\right\|_{L_{t}^{q} L_{x}^{r}\left(\left\{|t| \leq(3 M)^{-\frac{1}{\alpha}}\right\} \times \mathbb{R}^{d}\right)} \\
& \lesssim\left\||t|^{\alpha \beta}|\nabla|^{s_{c}+\beta+\gamma} P_{\geq 3 M}\left(\chi_{\geq \frac{1}{10} M|t|} e^{i t \Delta}\left(\chi_{\leq 10} P_{M} g\right)\right)\right\|_{L_{t}^{q} L_{x}^{r}\left(\left\{|t| \leq(3 M)^{-\frac{1}{\alpha}}\right\} \times \mathbb{R}^{d}\right)} \\
& \lesssim M^{-\beta}\left\||\nabla|^{s_{c}+\beta+\gamma} P_{\geq 3 M}\left(\chi_{\geq \frac{1}{10} M|t|} e^{i t \Delta}\left(\chi_{\leq 10} P_{M} g\right)\right)\right\|_{L_{t}^{q} L_{x}^{r}\left(\mathbb{R} \times \mathbb{R}^{d}\right)} .
\end{aligned}
$$


Then using Lemma 2.5 twice, Lemma 2.3 and Lemma 2.10, it is bounded by

$$
M^{-10}\left\|P_{M} g\right\|_{\dot{H}^{s_{c}}\left(\mathbb{R}^{d}\right)} .
$$

Therefore, we obtain

$$
(\underline{3.25 \mathrm{~b}}) \lesssim M^{-10}\left\|P_{M} g\right\|_{\dot{H}^{s_{c}}\left(\mathbb{R}^{d}\right)} .
$$

For the term (3.25c), we have

$$
\left(\underline{3.25 \mathrm{C})} \lesssim\left\|t^{\alpha \beta}|\nabla|^{s_{c}+\beta+\gamma} P_{\geq|t|-\alpha}\left(\chi_{\geq \frac{1}{10} M|t|} e^{i t \Delta}\left(\chi_{\leq 10} P_{M} g\right)\right)\right\|_{L_{t}^{q} L_{x}^{r}\left(\left\{|t| \geq(3 M)^{\left.-\frac{1}{\alpha}\right\} \times \mathbb{R}^{d}}\right)\right.} .\right.
$$

If $s_{c}+\beta+\gamma \geq 0$, using (3.20), (3.26) is bounded by

$$
M^{-(d-2)\left(\frac{1}{2}-\frac{1}{r}\right)+\beta+\gamma}\left\|t^{\alpha \beta-(d-1)\left(\frac{1}{2}-\frac{1}{r}\right)}\right\|_{L_{t}^{q}\left(\left\{|t| \geq(3 M)^{\left.\left.-\frac{1}{\alpha}\right\}\right)}\right.\right.}\left\|P_{M} g\right\|_{\dot{H}^{s_{c}}\left(\mathbb{R}^{d}\right)} .
$$

Using the condition of $(d-1)\left(\frac{1}{2}-\frac{1}{r}\right)>\alpha \beta+\frac{1}{q}$, it is dominated by

$$
M^{-(d-2)\left(\frac{1}{2}-\frac{1}{r}\right)+(d-1)\left(\frac{1}{2}-\frac{1}{r}\right) \cdot \frac{1}{\alpha}-\frac{1}{q \alpha}+\gamma}\left\|P_{M} g\right\|_{\dot{H}^{s_{c}}\left(\mathbb{R}^{d}\right)} .
$$

Now we claim that

$$
-(d-2)\left(\frac{1}{2}-\frac{1}{r}\right)+(d-1)\left(\frac{1}{2}-\frac{1}{r}\right) \cdot \frac{1}{\alpha}-\frac{1}{q \alpha}+\gamma \leq 0 .
$$

Indeed, using (2.5), the left-hand side of (3.28) is equal to

$$
\frac{2 \alpha-1}{\alpha} \cdot\left(\frac{1}{q}-(d-1)\left(\frac{1}{2}-\frac{1}{r}\right)\right) \text {. }
$$

Note that $\alpha \geq 1$, and from (3.23): $\frac{1}{q}<(d-1)\left(\frac{1}{2}-\frac{1}{r}\right)$, the last quantity above is negative. Hence, (3.28) is valid. Using (3.28), we have that (3.27) and thus (3.26) is bounded by $\left\|P_{M} g\right\|_{\dot{H}^{s c}\left(\mathbb{R}^{d}\right)}$.

If $s_{c}+\beta+\gamma<0$, using the Bernstein inequality, (3.26) is bounded by

$$
\begin{aligned}
\left\|t^{-\alpha\left(s_{c}+\gamma\right)}\left(\chi_{\geq \frac{1}{10} M|t|} e^{i t \Delta}\left(\chi_{\leq 10} P_{M} g\right)\right)\right\|_{L_{t}^{q} L_{x}^{r}\left(\left\{|t| \geq(3 M)^{-\frac{1}{\alpha}}\right\} \times \mathbb{R}^{d}\right)} \\
\quad \lesssim M^{-(d-2)\left(\frac{1}{2}-\frac{1}{r}\right)-s_{c}}\left\|t^{-\alpha\left(s_{c}+\gamma\right)-(d-1)\left(\frac{1}{2}-\frac{1}{r}\right)}\right\|_{L_{t}^{q}\left(\left\{|t| \geq(3 M)^{-\frac{1}{\alpha}}\right\}\right)}\left\|P_{M} g\right\|_{\dot{H}^{s_{c}}\left(\mathbb{R}^{d}\right)} .
\end{aligned}
$$

Then similarly as above, and using the condition of $(d-1)\left(\frac{1}{2}-\frac{1}{r}\right)+\alpha\left(s_{c}+\gamma\right)>\frac{1}{q}$, it is also bounded by

$$
M^{-(d-2)\left(\frac{1}{2}-\frac{1}{r}\right)+(d-1)\left(\frac{1}{2}-\frac{1}{r}\right) \cdot \frac{1}{\alpha}-\frac{1}{q \alpha}+\gamma}\left\|P_{M} g\right\|_{\dot{H}^{s^{c}}\left(\mathbb{R}^{d}\right)} .
$$

Hence, using (3.20) again, it is bounded by $\left\|P_{M} g\right\|_{\dot{H}^{s_{c}\left(\mathbb{R}^{d}\right)}}$. Therefore, we obtain that

$$
(\underline{3.25 \mathrm{c}}) \lesssim\left\|P_{M} g\right\|_{\dot{H}^{s_{c}}\left(\mathbb{R}^{d}\right)} .
$$

Combining the three estimates on (3.25), we get (3.24).

Together with Lemmas 3.3 and 3.5, Corollaries 3.4 and 3.6, we are ready to prove Proposition 3.1 . 
Proof of Proposition 3.1. Using Littlewood-Paley's decomposition, we have

$$
\begin{aligned}
& \left\||\nabla|^{s}\left(e^{i t \Delta}\left(\chi_{\leq 10} P_{\geq N} g\right)\right)\right\|_{L_{x}^{r}\left(\mathbb{R}^{d}\right)} \lesssim \sum_{M \geq N}\left\||\nabla|^{s}\left(e^{i t \Delta}\left(\chi_{\leq 10} P_{M} g\right)\right)\right\|_{L_{x}^{r}\left(\mathbb{R}^{d}\right)} \\
& \quad \lesssim \sum_{M \geq N}\left\||\nabla|^{s}\left(\chi_{\leq \frac{1}{10} M|t|} e^{i t \Delta}\left(\chi_{\leq 10} P_{M} g\right)\right)\right\|_{L_{x}^{r}\left(\mathbb{R}^{d}\right)}+\sum_{M \geq N}\left\||\nabla|^{s}\left(\chi_{\geq \frac{1}{10} M|t|} e^{i t \Delta}\left(\chi_{\leq 10} P_{M} g\right)\right)\right\|_{L_{x}^{r}\left(\mathbb{R}^{d}\right)} .
\end{aligned}
$$

Using Lemma 3.3, we get that for any $K \in \mathbb{Z}^{+}$,

$$
\sum_{M \geq N}\left\||\nabla|^{s}\left(\chi_{\leq \frac{1}{10} M|t|} e^{i t \Delta}\left(\chi_{\leq 10} P_{M} g\right)\right)\right\|_{L_{x}^{r}\left(\mathbb{R}^{d}\right)} \lesssim|t|^{-d\left(\frac{1}{2}-\frac{1}{r}\right)} N^{-K}\left\|P_{\geq N} g\right\|_{\dot{H}^{s_{c}}\left(\mathbb{R}^{d}\right)}
$$

Using Lemma 3.5,

$$
\sum_{M \geq N}\left\||\nabla|^{s}\left(\chi_{\geq \frac{1}{10} M|t|} e^{i t \Delta}\left(\chi_{\leq 10} P_{M} g\right)\right)\right\|_{L_{x}^{r}\left(\mathbb{R}^{d}\right)} \lesssim N^{-(d-2)\left(\frac{1}{2}-\frac{1}{r}\right)+s-s_{c}}|t|^{-(d-1)\left(\frac{1}{2}-\frac{1}{r}\right)}\left\|P_{\geq N} g\right\|_{\dot{H}_{x}^{s_{c}}\left(\mathbb{R}^{d}\right)} .
$$

Combining these estimates, we obtain (3.1).

Now we prove (3.3). Firstly, we give a reduction as following. Fix $q \geq 2$, and let $\varepsilon_{0}=\frac{1}{100 q}$. Then to prove (3.3), we only need to consider the estimates on the triples $(q, r, \gamma)$ when $\gamma \geq \varepsilon_{0}$. Indeed, if $\gamma \leq \varepsilon_{0}$, then by the Sobolev inequality it follows from the case when $r$ and $\gamma$ satisfying

$$
\frac{1}{r}=\frac{1}{2}-\frac{2}{d q}+\frac{\varepsilon_{0}}{d} ; \quad \gamma=\varepsilon_{0}
$$

Using Littlewood-Paley's decomposition and Lemma 2.4,

$$
\begin{aligned}
& \left\|\left\langle t^{\alpha}|\nabla|\right\rangle^{\beta}|\nabla|^{s_{c}+\gamma} e^{i t \Delta}\left(\chi_{\leq 10} P_{\geq N} g\right)\right\|_{L_{t}^{q} L_{x}^{r}\left(\mathbb{R} \times \mathbb{R}^{d}\right)} \\
& \lesssim\left\|\left\langle t^{\alpha}|\nabla|\right\rangle^{\beta}|\nabla|^{s_{c}+\gamma} e^{i t \Delta}\left(\chi_{\leq 10} \sum_{M \geq N} P_{M} g\right)\right\|_{L_{t}^{q} L_{x}^{r}\left(\mathbb{R} \times \mathbb{R}^{d}\right)} \\
& \quad \lesssim\left\|\left(\sum_{M \geq N}\left(\left\langle t^{\alpha}|\nabla|\right\rangle^{\beta}|\nabla|^{s_{c}+\gamma} e^{i t \Delta}\left(\chi_{\leq 10} P_{M} g\right)\right)^{2}\right)^{\frac{1}{2}}\right\|_{L_{t}^{q} L_{x}^{r}\left(\mathbb{R} \times \mathbb{R}^{d}\right)}
\end{aligned}
$$

Since $q \geq 2, r \geq 2$, it is dominated by

$$
\left(\sum_{M \geq N}\left\|\left\langle t^{\alpha}|\nabla|\right\rangle^{\beta}|\nabla|^{s_{c}+\gamma} e^{i t \Delta}\left(\chi_{\leq 10} P_{M} g\right)\right\|_{L_{t}^{q} L_{x}^{r}\left(\mathbb{R} \times \mathbb{R}^{d}\right)}^{2}\right)^{\frac{1}{2}} .
$$

Therefore, we obtain

$$
\begin{aligned}
& \left\|\left\langle t^{\alpha}|\nabla|\right\rangle^{\beta}|\nabla|^{s_{c}+\gamma}\left(e^{i t \Delta}\left(\chi_{\leq 10} P_{\geq N} g\right)\right)\right\|_{L_{t}^{q} L_{x}^{r}\left(\mathbb{R} \times \mathbb{R}^{d}\right)} \\
& \lesssim\left(\sum_{M \geq N}\left\|\left\langle t^{\alpha}|\nabla|\right\rangle^{\beta}|\nabla|^{s_{c}+\gamma} e^{i t \Delta}\left(\chi_{\leq 10} P_{M} g\right)\right\|_{L_{t}^{q} L_{x}^{r}\left(\mathbb{R} \times \mathbb{R}^{d}\right)}^{2}\right)^{\frac{1}{2}} .
\end{aligned}
$$

Now we check the conditions (3.14) and (3.23). Setting

$$
s_{*}=\max \left\{-\varepsilon_{0}, s_{*, 1}, s_{*, 2}\right\},
$$

then $s_{c}+\gamma \geq 0$. Hence, the conditions (3.14) and (3.23) reduce to

$$
(d-1)\left(\frac{1}{2}-\frac{1}{r}\right)>\alpha \beta+\frac{1}{q}
$$


which is valid by choosing $\alpha \beta$ small enough. Then by Corollaries 3.4 and 3.6, we have

$$
\begin{aligned}
\|\left\langle t^{\alpha}|\nabla|\right\rangle^{\beta}|\nabla|^{s_{c}+\gamma}\left(e^{i t \Delta}\left(\chi_{\leq 10} P_{\geq N} g\right)\right) & \|_{L_{t}^{q} L_{x}^{r}\left(\mathbb{R} \times \mathbb{R}^{d}\right)} \\
\lesssim & \left(\sum_{M \geq N}\left\|P_{M} g\right\|_{\dot{H}^{s_{c}}\left(\mathbb{R}^{d}\right)}^{2}\right)^{\frac{1}{2}} \lesssim\left\|P_{\geq N} g\right\|_{\dot{H}^{s_{c}}\left(\mathbb{R}^{d}\right)} .
\end{aligned}
$$

This proves the proposition.

\section{Nonlinear Flow estimates on LOCAlized initial Data}

In this section, we give some nonlinear estimates. Firstly, we give some local time and small data estimates.

4.1. Local theory. Since $u_{0} \in \dot{H}^{s_{c}}\left(\mathbb{R}^{d}\right)$, we have the following local and small data results, the proofs are standard. However, we give the details for the sake of the completeness. The first is essentially proved by Guo, Wang [28].

Lemma 4.1. Let $s_{0}=-\min \left\{\frac{d-1}{2 d-1}, \frac{2(d-1)}{(2 d-1)(p+1)}\right\}$, then for any $s_{c}>s_{0}$, the following result holds. For any fixing $\delta_{0}>0$, and any radial function $u_{0} \in \dot{H}^{s_{c}}\left(\mathbb{R}^{d}\right)$, there exists $t_{0}=$ $t_{0}\left(u_{0}, \delta_{0}\right)>0$, such that the Cauchy problem (1.1) is well-posed on the time interval $\left[0, t_{0}\right]$. Moreover the solution u satisfies

$$
\|u\|_{L_{t}^{\infty} \dot{H}_{x}^{s_{c}}\left(\left[0, t_{0}\right] \times \mathbb{R}^{d}\right)} \lesssim 1 ; \quad\left\||\nabla|^{s_{c}+\gamma} u\right\|_{L_{t}^{q} L_{x}^{r}\left(\left[0, t_{0}\right] \times \mathbb{R}^{d}\right)} \lesssim \delta_{0}
$$

Here the triple $(q, r, \gamma)$ verifies (2.5) and $\gamma \in\left[-s_{c},-s_{0}\right)$.

Remark 4.2. The result in this lemma improves the index obtained by Guo, Wang [28], who proved the local well-posedness in $\dot{H}^{s_{c}}\left(\mathbb{R}^{d}\right)$ when $s_{c}>-\frac{d-1}{2 d+1}$ for radial datum. In particular, in this lemma, when $d \geq 4$, the restriction is $s_{c}>-\frac{d-1}{2 d-1}\left(s_{c}>-0.275,-0.388\right.$ when $d=2,3$ respectively).

Proof of Lemma 4.1. We only show (4.1) for some $t_{0}>0$. Then the local well-posedness with the lifespan $\left[0, t_{0}\right)$ is followed by the standard fixed point argument. In the following, we prove (4.1) by two cases: $p \leq 1$ and $p>1$ separately.

If $p \leq 1$, we denote the parameter $r_{1}$ as

$$
\frac{1}{r_{1}}=\frac{1}{2}-\frac{1}{d}+\frac{\gamma}{d}
$$

Then for any $s_{c}>-\frac{d-1}{2 d-1}$ and $\gamma \geq-s_{c}$, by the Duhamel formula and Lemma 2.10, we have

$$
\left\||\nabla|^{s_{c}+\gamma} u\right\|_{L_{t}^{2} L_{x}^{r_{1}}\left(\left[0, t_{0}\right] \times \mathbb{R}^{d}\right)} \lesssim\left\||\nabla|^{s_{c}+\gamma} e^{i t \Delta} u_{0}\right\|_{L_{t}^{2} L_{x}^{r_{1}\left(\left[0, t_{0}\right] \times \mathbb{R}^{d}\right)}}+\left\||\nabla|^{s_{c}+\gamma}\left(|u|^{p} u\right)\right\|_{L_{t}^{\tilde{q}^{\prime}} L_{x}^{\tilde{r}^{\prime}}\left(\left[0, t_{0}\right] \times \mathbb{R}^{d}\right)},
$$

where $(\tilde{q}, \tilde{r})$ satisfies

Hence, by Lemma 2.2, we get

$$
\tilde{q}=\frac{2}{1-p}, \quad \frac{2}{\tilde{q}}+\frac{d}{\tilde{r}}=\frac{d}{2}-\gamma
$$

$$
\begin{aligned}
& \left\||\nabla|^{s_{c}+\gamma} u\right\|_{L_{t}^{2} L_{x}^{r_{1}}\left(\left[0, t_{0}\right] \times \mathbb{R}^{d}\right)} \lesssim\left\||\nabla|^{s_{c}+\gamma} e^{i t \Delta} u_{0}\right\|_{L_{t}^{2} L_{x}^{r_{1}}\left(\left[0, t_{0}\right] \times \mathbb{R}^{d}\right)} \\
& +\left\||\nabla|^{s_{c}+\gamma} u\right\|_{L_{t}^{2} L_{x}^{r_{1}}\left(\left[0, t_{0}\right] \times \mathbb{R}^{d}\right)}\|u\|_{L_{t}^{2} L_{x}^{r_{2}}\left(\left[0, t_{0}\right] \times \mathbb{R}^{d}\right)}^{p},
\end{aligned}
$$


where the parameter $r_{2}$ satisfies

$$
\frac{1}{r_{2}}=\frac{1}{2}-\frac{1}{d}-\frac{s_{c}}{d}=\frac{2-p}{d p} .
$$

Then by the Sobolev inequality, we obtain that

$$
\left\||\nabla|^{s_{c}+\gamma} u\right\|_{L_{t}^{2} L_{x}^{r_{1}}\left(\left[0, t_{0}\right] \times \mathbb{R}^{d}\right)} \lesssim\left\||\nabla|^{s_{c}+\gamma} e^{i t \Delta} u_{0}\right\|_{L_{t}^{2} L_{x}^{r_{1}}\left(\left[0, t_{0}\right] \times \mathbb{R}^{d}\right)}+\left\||\nabla|^{s_{c}+\gamma} u\right\|_{L_{t}^{2} L_{x}^{r_{1}}\left(\left[0, t_{0}\right] \times \mathbb{R}^{d}\right)}^{p+1} .
$$

Therefore, for any $\delta_{0}>0$, if

$$
\left\||\nabla|^{s_{c}+\gamma} e^{i t \Delta} u_{0}\right\|_{L_{t}^{2} L_{x}^{r_{1}}\left(\left[0, t_{0}\right] \times \mathbb{R}^{d}\right)} \leq \delta_{0},
$$

then by the continuity argument,

$$
\left\||\nabla|^{s_{c}+\gamma} u\right\|_{L_{t}^{2} L_{x}^{r_{1}\left(\left[0, t_{0}\right] \times \mathbb{R}^{d}\right)}} \lesssim \delta_{0} .
$$

Note that

$$
\left\||\nabla|^{s_{c}+\gamma} e^{i t \Delta} u_{0}\right\|_{L_{t}^{2} L_{x}^{r_{1}}\left(\mathbb{R} \times \mathbb{R}^{d}\right)} \lesssim\left\|u_{0}\right\|_{\dot{H}^{s_{c}}\left(\mathbb{R}^{d}\right)}
$$

(4.2) is verified when $t_{0}=t_{0}\left(u_{0}, \delta_{0}\right)$ is small enough, and thus we have (4.3).

Similarly,

$$
\begin{aligned}
\|u\|_{L_{t}^{\infty} \dot{H}_{x}^{s_{c}}\left(\left[0, t_{0}\right] \times \mathbb{R}^{d}\right)} & \lesssim\left\|e^{i t \Delta} u_{0}\right\|_{L_{t}^{\infty} \dot{H}_{x}^{s_{c}}\left(\left[0, t_{0}\right] \times \mathbb{R}^{d}\right)}+\left\||\nabla|^{s_{c}+\gamma}\left(|u|^{p} u\right)\right\|_{L_{t}^{\tilde{q}^{\prime}} L_{x}^{\tilde{r}^{\prime}}\left(\left[0, t_{0}\right] \times \mathbb{R}^{d}\right)} \\
& \lesssim\left\|u_{0}\right\|_{\dot{H}^{s_{c}}\left(\mathbb{R}^{d}\right)}+\left\|\left.\nabla\right|^{s_{c}+\gamma} u\right\|_{L_{t}^{2} L_{x}^{r_{1}\left(\left[0, t_{0}\right] \times \mathbb{R}^{d}\right)}}^{p} .
\end{aligned}
$$

Then by (4.3), we obtain that

$$
\|u\|_{L_{t}^{\infty} \dot{H}_{x}^{s_{c}}\left(\left[0, t_{0}\right] \times \mathbb{R}^{d}\right)} \lesssim 1 .
$$

Further, for general triple $(q, r, \gamma)$ verifying (2.5) and $\gamma \in\left[-s_{c}, \frac{d-1}{2 d-1}\right)$,

$$
\begin{aligned}
\left\||\nabla|^{s_{c}+\gamma} u\right\|_{L_{t}^{q} L_{x}^{r}\left(\left[0, t_{0}\right] \times \mathbb{R}^{d}\right)} & \lesssim\left\||\nabla|^{s_{c}+\gamma} e^{i t \Delta} u_{0}\right\|_{L_{t}^{q} L_{x}^{r}\left(\left[0, t_{0}\right] \times \mathbb{R}^{d}\right)}+\left\||\nabla|^{s_{c}+\gamma}\left(|u|^{p} u\right)\right\|_{L_{t}^{\tilde{q}^{\prime}} L_{x}^{\tilde{r}^{\prime}}\left(\left[0, t_{0}\right] \times \mathbb{R}^{d}\right)} \\
& \lesssim \delta_{0}+\left\||\nabla|^{s_{c}+\gamma} u\right\|_{L_{t}^{2} L_{x}^{r_{1}}\left(\left[0, t_{0}\right] \times \mathbb{R}^{d}\right)} .
\end{aligned}
$$

Hence, we get

$$
\left\||\nabla|^{s_{c}+\gamma} u\right\|_{L_{t}^{q} L_{x}^{r}\left(\left[0, t_{0}\right] \times \mathbb{R}^{d}\right)} \lesssim \delta_{0}
$$

If $p>1$, we denote the parameter $r_{3}$ as

$$
\frac{1}{r_{3}}=\frac{1}{2}-\frac{2}{d(p+1)}+\frac{\gamma}{d}
$$

Then similarly as above, we obtain that for any $s_{c}>-\frac{2(d-1)}{(2 d-1)(p+1)}$ and $\gamma \geq-s_{c}$, $\left\||\nabla|^{s_{c}+\gamma} u\right\|_{L_{t}^{p+1} L_{x}^{r_{3}}\left(\left[0, t_{0}\right] \times \mathbb{R}^{d}\right)} \lesssim\left\||\nabla|^{s_{c}+\gamma} e^{i t \Delta} u_{0}\right\|_{L_{t}^{p+1} L_{x}^{r_{3}}\left(\left[0, t_{0}\right] \times \mathbb{R}^{d}\right)}+\left\||\nabla|^{s_{c}+\gamma}\left(|u|^{p} u\right)\right\|_{L_{t}^{1} L_{x}^{r_{4}^{\prime}\left(\left[0, t_{0}\right] \times \mathbb{R}^{d}\right)}}$, where $r_{4}$ satisfies

$$
\frac{d}{r_{4}}=\frac{d}{2}-\gamma
$$

Hence, by Lemma 2.2 and Sobolev's inequality, we get

$$
\left\||\nabla|^{s_{c}+\gamma} u\right\|_{L_{t}^{p+1} L_{x}^{r_{3}\left(\left[0, t_{0}\right] \times \mathbb{R}^{d}\right)}} \lesssim\left\||\nabla|^{s_{c}+\gamma} e^{i t \Delta} u_{0}\right\|_{L_{t}^{p+1} L_{x}^{r_{3}}\left(\left[0, t_{0}\right] \times \mathbb{R}^{d}\right)}+\left\||\nabla|^{s_{c}+\gamma} u\right\|_{L_{t}^{p+1} L_{x}^{r_{3}}\left(\left[0, t_{0}\right] \times \mathbb{R}^{d}\right)}^{p+1} .
$$

Treating similarly as above, by choosing $t_{0}=t_{0}\left(u_{0}, \delta_{0}\right)$ small enough, we obtain that

$$
\left\||\nabla|^{s_{c}+\gamma} u\right\|_{L_{t}^{p+1} L_{x}^{r_{3}}\left(\left[0, t_{0}\right] \times \mathbb{R}^{d}\right)} \lesssim \delta_{0},
$$


and thus obtain (4.1).

Now we fix $\delta_{0} \in(0,1)$ to be an absolute small constant which will determined later. For simplicity, we set $t_{0}\left(u_{0}, \delta_{0}\right)=2$ by rescaling. Moreover, we set a number $N=N\left(\delta_{0}\right)$ such that

$$
\left\|P_{\geq N} u_{0}\right\|_{\dot{H}^{s_{c}\left(\mathbb{R}^{d}\right)}} \leq \delta_{0} .
$$

To prove Theorem 1.1, we split the initial data $u_{0}$ into three parts as

$$
u_{0}=\chi_{\leq 10}\left(P_{\geq N} u_{0}\right)+P_{\leq N} u_{0}+\chi_{\geq 10}\left(P_{\geq N} u_{0}\right) .
$$

Accordingly, let

$$
v_{0}=\chi_{\leq 10}\left(P_{\geq N} u_{0}\right),
$$

and $v$ be the solution of the following equation,

$$
\left\{\begin{array}{l}
i \partial_{t} v+\Delta v=\chi_{\leq 1}(t)|v|^{p} v \\
v(0, x)=v_{0}
\end{array}\right.
$$

Moreover, let

$$
w_{0}=\chi_{\geq 10}\left(P_{\geq N} u_{0}\right)+P_{\leq N} u_{0},
$$

and $w=u-v$. Then $w$ is the solution of the following equation,

$$
\left\{\begin{array}{l}
i \partial_{t} w+\Delta w=|u|^{p} u-\chi_{\leq 1}(t)|v|^{p} v, \\
w(0, x)=w_{0} .
\end{array}\right.
$$

Then the second result is a global result with small data in $\dot{H}^{s_{c}}$-level.

Lemma 4.3. For any $s_{c}>s_{0}$, the following result holds. Let $u_{0} \in \dot{H}^{s_{c}}\left(\mathbb{R}^{d}\right)$ be radial, then there exist a small constant $\delta_{0}$ and a large constant $N$ verifying (4.4), such that the Cauchy problem (4.5) is globally well-posed. In particular, the solution v satisfies

$$
\|v\|_{L_{t}^{\infty} \dot{H}_{x}^{s_{c}}\left(\mathbb{R} \times \mathbb{R}^{d}\right)}+\left\||\nabla|^{s_{c}+\gamma} v\right\|_{L_{t}^{q} L_{x}^{r}\left(\mathbb{R} \times \mathbb{R}^{d}\right)} \lesssim\left\|v_{0}\right\|_{\dot{H}^{s_{c}}\left(\mathbb{R}^{d}\right)} .
$$

Here the triple $(q, r, \gamma)$ verifies (2.5) and $\gamma \in\left[0,-s_{0}\right)$.

Proof. We adopt the same notation and argue similarly as in the proof of Lemma 4.1. Moreover, we may assume that $\gamma \geq-s_{c}$, otherwise it follows by the Sobolev inequality. In the case of $p \leq 1$, for any $s_{c}>-\frac{\bar{d}-1}{2 d-1}$,

$$
\left\||\nabla|^{s_{c}+\gamma} v\right\|_{L_{t}^{2} L_{x}^{r_{1}\left(\mathbb{R} \times \mathbb{R}^{d}\right)}} \lesssim\left\|v_{0}\right\|_{\dot{H}^{s_{c}\left(\mathbb{R}^{d}\right)}}+\left\||\nabla|^{s_{c}+\gamma} v\right\|_{L_{t}^{2} L_{x}^{r_{1}\left(\mathbb{R} \times \mathbb{R}^{d}\right)}}^{p+1} .
$$

Hence, by the continuity argument and choosing $\delta_{0}$ small enough, we obtain

$$
\left\||\nabla|^{s_{c}+\gamma} v\right\|_{L_{t}^{2} L_{x}^{r_{1}}\left(\mathbb{R} \times \mathbb{R}^{d}\right)} \lesssim\left\|v_{0}\right\|_{\dot{H}^{s_{c}\left(\mathbb{R}^{d}\right)}} .
$$

Using the estimate above, we have the desired results. In the case of $p>1$, for any $s_{c}>$ $-\frac{2(d-1)}{(2 d-1)(p+1)}$

$$
\left\||\nabla|^{s_{c}+\gamma} v\right\|_{L_{t}^{p+1} L_{x}^{r_{3}}\left(\mathbb{R} \times \mathbb{R}^{d}\right)} \lesssim\left\|v_{0}\right\|_{\dot{H}^{s_{c}}\left(\mathbb{R}^{d}\right)}+\left\||\nabla|^{s_{c}+\gamma} v\right\|_{L_{t}^{p+1} L_{x}^{r_{3}}\left(\mathbb{R} \times \mathbb{R}^{d}\right)}^{p+1} .
$$

Hence, arguing similarly as above, we obtain the desired estimates again. 
4.2. Nonlinear estimates on $v$. In this subsection, we give the estimates on $v$. For convenience, we introduce some notation. We denote $X(\alpha, \beta)$ be the space with the norm:

$$
\|f\|_{X(\alpha, \beta)}=\left\|\left\langle t^{\alpha}|\nabla|\right\rangle^{\beta}|\nabla|^{s_{c}} P_{M} f\right\|_{l_{M}^{\infty} L_{t}^{2} L_{x}^{\frac{2 d}{d-2}}\left(\{M \geq 1\} \times \mathbb{R} \times \mathbb{R}^{d}\right)} .
$$

Then the main result in this subsection is

Proposition 4.4. Let $v$ be the solution of (4.5), then there exist $\alpha_{0} \geq 1, \beta_{0}>0$ and $s_{*}<0$, such that for any $s_{c} \in\left[s_{*}, 0\right)$,

$$
\|v\|_{X\left(\alpha_{0}, \beta_{0}\right)} \lesssim\left\|v_{0}\right\|_{\dot{H}^{s_{c}}\left(\mathbb{R}^{d}\right)}
$$

Proof. We write

$$
\begin{aligned}
& \left\|\left\langle t^{\alpha_{0}}|\nabla|\right\rangle^{\beta_{0}}|\nabla|^{s_{c}} P_{M} v\right\|_{l_{M}^{\infty} L_{t}^{2} L_{x}^{\frac{2 d}{d-2}}\left(\{M \geq 1\} \times \mathbb{R} \times \mathbb{R}^{d}\right)} \\
& =\left\|\left\langle t^{\alpha_{0}}|\nabla|\right\rangle^{\beta_{0}}|\nabla|^{s_{c}} P_{M} v\right\|_{l_{M}^{\infty} L_{t}^{2} L_{x}^{\frac{2 d}{d-2}}\left(\{M \geq 1\} \times\left\{|t| \leq M^{-\frac{1}{\alpha_{0}}}\right\} \times \mathbb{R}^{d}\right)} \\
& \quad+\left\|\left\langle t^{\alpha_{0}}|\nabla|\right\rangle^{\beta_{0}}|\nabla|^{s_{c}} P_{M} v\right\|_{l_{M}^{\infty} L_{t}^{2} L_{x}^{\frac{2 d}{d-2}}\left(\{M \geq 1\} \times\left\{|t| \geq M^{-\frac{1}{\alpha_{0}}}\right\} \times \mathbb{R}^{d}\right)} .
\end{aligned}
$$

Estimates on (4.7). Note that

$$
\left\|\left\langle t^{\alpha_{0}}|\nabla|\right\rangle^{\beta_{0}}|\nabla|^{s_{c}} P_{M} v\right\|_{L_{t}^{2} L_{x}^{\frac{2 d}{d-2}}\left(\left\{|t| \leq M^{\left.\left.-\frac{1}{\alpha_{0}}\right\} \times \mathbb{R}^{d}\right)}\right.\right.} \lesssim\left\||\nabla|^{s_{c}} P_{M} v\right\|_{L_{t}^{2} L_{x}^{\frac{2 d}{d-2}}\left(\mathbb{R} \times \mathbb{R}^{d}\right)} .
$$

Then by Lemma 4.3 (where we choose the triple $(q, r, \gamma)=\left(2, \frac{2 d}{d-2}, 0\right)$ ), it is further controlled by $\left\|v_{0}\right\|_{\dot{H}^{s_{c}\left(\mathbb{R}^{d}\right)}}$. Therefore, we have the bound of (4.7) as

$$
\left\|\left\langle t^{\alpha_{0}}|\nabla|\right\rangle^{\beta_{0}}|\nabla|^{s_{c}} P_{M} v\right\|_{l_{M}^{\infty} L_{t}^{2} L_{x}^{\frac{2 d}{d-2}}\left(\{M \geq 1\} \times\left\{|t| \leq M^{\left.\left.-\frac{1}{\alpha_{0}}\right\} \times \mathbb{R}^{d}\right)}\right.\right.} \lesssim\left\|v_{0}\right\|_{\dot{H}^{s_{c}}\left(\mathbb{R}^{d}\right)} .
$$

Estimates on (4.8). It is controlled by

$$
\left\|t^{\alpha_{0} \beta_{0}}|\nabla|^{\beta_{0}+s_{c}} P_{M} v\right\|_{l_{M}^{\infty} L_{t}^{2} L_{x}^{\frac{2 d}{d-2}}\left(\{M \geq 1\} \times\left\{|t| \geq M^{-\frac{1}{\alpha_{0}}}\right\} \times \mathbb{R}^{d}\right)} .
$$

We only consider the positive time, that is, $t \geq 0$, the negative time being obtained in the same way. Now we write

$$
\begin{aligned}
P_{M} v=e^{i t \Delta} P_{M} v_{0} & +\int_{0}^{\frac{1}{2} t} e^{i(t-s) \Delta} \chi_{\leq 1}(s) P_{M}\left(|v|^{p} v\right) d s \\
& +\int_{\frac{1}{2} t}^{t} e^{i(t-s) \Delta} \chi_{\leq 1}(s) P_{M}\left(|v|^{p} v\right) d s,
\end{aligned}
$$

then we need to consider the following three parts,

$$
\begin{aligned}
& \left\|t^{\alpha_{0} \beta_{0}}|\nabla|^{\beta_{0}+s_{c}} e^{i t \Delta} P_{M} v_{0}\right\|_{l_{M}^{\infty} L_{t}^{2} L_{x}^{\frac{2 d}{d-2}}\left(\{M \geq 1\} \times\left\{|t| \geq M^{-\frac{1}{\alpha_{0}}}\right\} \times \mathbb{R}^{d}\right)} ; \\
& \left\|t^{\alpha_{0} \beta_{0}}|\nabla|^{\beta_{0}+s_{c}} \int_{0}^{\frac{1}{2} t} e^{i(t-s) \Delta} \chi_{\leq 1}(s) P_{M}\left(|v|^{p} v\right) d s\right\|_{l_{M}^{\infty} L_{t}^{2} L_{x}^{\frac{2 d}{d-2}}\left(\{M \geq 1\} \times\left\{|t| \geq M^{-\frac{1}{\alpha_{0}}}\right\} \times \mathbb{R}^{d}\right)} ;
\end{aligned}
$$

and

$$
\left\|t^{\alpha_{0} \beta_{0}}|\nabla|^{\beta_{0}+s_{c}} \int_{\frac{1}{2} t}^{t} e^{i(t-s) \Delta} \chi_{\leq 1}(s) P_{M}\left(|v|^{p} v\right) d s\right\|_{l_{M}^{\infty} L_{t}^{2} L_{x}^{\frac{2 d}{d-2}}\left(\{M \geq 1\} \times\left\{|t| \geq M^{-\frac{1}{\alpha_{0}}}\right\} \times \mathbb{R}^{d}\right)} .
$$


Estimates on (4.10a). Here we choose $s_{*}=s_{*}(2), \alpha_{0} \geq 1$ and $\beta_{0}>0$ with $\alpha \beta \leq$ $\eta_{*}\left(2, \frac{2 d}{d-2}, 0\right)$, where $s_{*}, \eta_{*}$ are the parameters obtained in Proposition 3.1 (we may narrow $s_{*}$ suitably in the following if necessary). Then by Proposition 3.1, we obtain that for any $s_{c} \geq s_{*}$,

$$
\left\|t^{\alpha_{0} \beta_{0}}|\nabla|^{\beta_{0}+s_{c}} e^{i t \Delta} P_{M} v_{0}\right\|_{l_{M}^{\infty} L_{t}^{2} L_{x}^{\frac{2 d}{d-2}}\left(\{M \geq 1\} \times\left\{|t| \geq M^{-\frac{1}{\alpha_{0}}}\right\} \times \mathbb{R}^{d}\right)} \lesssim\left\|v_{0}\right\|_{\dot{H}^{s_{c}}\left(\mathbb{R}^{d}\right)} .
$$

Estimates on (4.10b). From Lemma 2.8.

$$
\begin{aligned}
\| t^{\alpha_{0} \beta_{0}} \mid & \left.\nabla\right|^{\beta_{0}+s_{c}} \int_{0}^{\frac{1}{2} t} e^{i(t-s) \Delta} \chi_{\leq 1}(s) P_{M}\left(|v|^{p} v\right) d s \|_{L_{t}^{2} L_{x}^{\frac{2 d}{d-2}}\left(\left\{|t| \geq M^{-\frac{1}{\alpha_{0}}}\right\} \times \mathbb{R}^{d}\right)} \\
& \lesssim\left\|t^{\alpha_{0} \beta_{0}} \int_{0}^{\frac{1}{2} t}|t-s|^{-1} \chi_{\leq 1}(s)\right\||\nabla|^{\beta_{0}+s_{c}} P_{M}\left(|v|^{p} v\right)\left\|_{L^{\frac{2 d}{d+2}}\left(\mathbb{R}^{d}\right)} d s\right\|_{L_{t}^{2}\left(\left\{|t| \geq M^{-\frac{1}{\alpha_{0}}}\right\}\right)} \\
& \lesssim\left\|t^{\alpha_{0} \beta_{0}-1} \int_{0}^{2}\right\||\nabla|^{\beta_{0}+s_{c}} P_{M}\left(|v|^{p} v\right)\left\|_{L_{x}^{\frac{2 d}{d+2}}\left(\mathbb{R}^{d}\right)} d s\right\|_{L_{t}^{2}\left(\left\{|t| \geq M^{\left.\left.-\frac{1}{\alpha_{0}}\right\}\right)}\right.\right.},
\end{aligned}
$$

where we have used the relationship $|t-s| \sim|t|$. We can choose $\alpha_{0} \beta_{0}$ small enough, such that $\alpha_{0} \beta_{0}<\frac{1}{2}$. Then taking $L_{t}^{2}$ first and using Bernstein's inequality, the inequality above is bounded by

$$
\int_{0}^{2}\left\||\nabla|^{s_{c}+\frac{1}{2 \alpha_{0}}} P_{M}\left(|v|^{p} v\right)\right\|_{L_{x}^{\frac{2 d}{d+2}}\left(\mathbb{R}^{d}\right)} d s
$$

Now we consider the following two cases. The first case is $s_{c}+\frac{1}{2 \alpha_{0}} \leq 0$. Then (4.11) is dominated by

$$
\int_{0}^{2}\left\||v|^{p} v\right\|_{L_{x}^{\frac{2 d}{d+2}}\left(\mathbb{R}^{d}\right)} d s
$$

Using the Hölder inequality, it is further controlled by

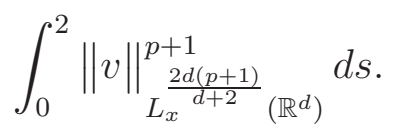

Let $q_{1}$ verify

$$
\frac{1}{q_{1}}=\frac{1}{p}-\frac{d+2}{4(p+1)} .
$$

Then $\left(q_{1}, \frac{2 d(p+1)}{d+2},-s_{c}\right)$ verifies (2.5) (decreasing the distance between $p_{0}(d)$ and $\frac{4}{d}$ to satisfy the conditions in (2.5) if necessary).

Note that $q_{1} \geq p+1$ when $s_{*}$ is close enough to zero (indeed, if $s_{c}=0$, then $q_{1}=2(p+1)$ ), and thus (4.12) is bounded by

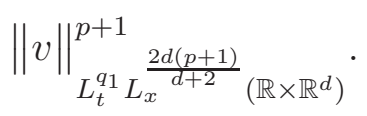

Using Lemma 4.3, it is bounded again by $\left\|v_{0}\right\|_{\dot{H}^{s_{c}}\left(\mathbb{R}^{d}\right)}^{p+1}$. Hence, we obtain

$$
\left\|t^{\alpha_{0} \beta_{0}}|\nabla|^{\beta_{0}+s_{c}} \int_{0}^{\frac{1}{2} t} e^{i(t-s) \Delta} \chi_{\leq 1}(s) P_{M}\left(|v|^{p} v\right) d s\right\|_{L_{t}^{2} L_{x}^{\frac{2 d}{d-2}}\left(\left\{|t| \geq M^{\left.\left.-\frac{1}{\alpha_{0}}\right\} \times \mathbb{R}^{d}\right)}\right.\right.} \lesssim\left\|v_{0}\right\|_{\dot{H}^{s c}\left(\mathbb{R}^{d}\right)}^{p+1} .
$$

The second case is $s_{c}+\frac{1}{2 \alpha_{0}}>0$. Then (4.11) is bounded by

$$
\int_{0}^{2}\left\||\nabla|^{s_{c}+\frac{1}{2 \alpha_{0}}}\left(|v|^{p} v\right)\right\|_{L_{x}^{\frac{2 d}{d+2}}\left(\mathbb{R}^{d}\right)} d s .
$$


Then using Lemma 2.2 and the Hölder inequality, it is further controlled by

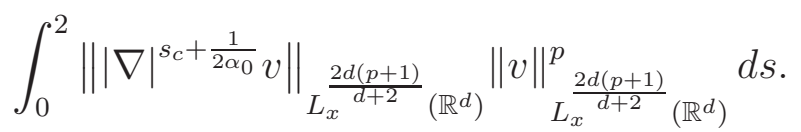

Let $q_{2}$ verify

$$
\frac{1}{q_{2}}=\frac{d}{4}+\frac{1}{4 \alpha_{0}}-\frac{d+2}{4(p+1)}
$$

then for suitable large $\alpha_{0}$ and small $\left|s_{*}\right|,\left(q_{2}, \frac{2 d(p+1)}{d+2},-\frac{1}{2 \alpha_{0}}\right)$ verifies (2.5). Moreover, we have

$$
\frac{1}{q_{2}}+\frac{p}{q_{1}} \leq 1
$$

(In particular, if $s_{c}=0, \alpha_{0}=+\infty$, then $q_{1}=q_{2}=2(p+1)$, hence the conclusions verify when we choose $\left|s_{*}\right|$ small enough and $\alpha_{0}$ large enough). Hence, (4.13) is bounded by

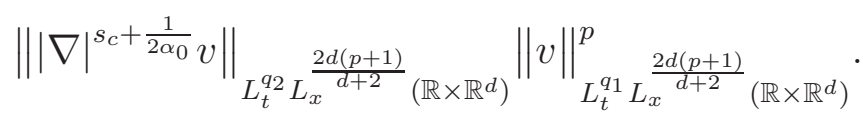

Using Lemma 4.3 again, it is bounded by $\left\|v_{0}\right\|_{\dot{H}^{s_{c}\left(\mathbb{R}^{d}\right)}}^{p+1}$. Hence, we also obtain

$$
\left\|t^{\alpha_{0} \beta_{0}}|\nabla|^{\beta_{0}+s_{c}} \int_{0}^{\frac{1}{2} t} e^{i(t-s) \Delta} \chi_{\leq 1}(s) P_{M}\left(|v|^{p} v\right) d s\right\|_{L_{t}^{2} L_{x}^{\frac{2 d}{d-2}}\left(\left\{|t| \geq M^{\left.\left.-\frac{1}{\alpha_{0}}\right\} \times \mathbb{R}^{d}\right)}\right.\right.} \lesssim\left\|v_{0}\right\|_{\dot{H}^{s_{c}\left(\mathbb{R}^{d}\right)}}^{p+1} .
$$

Therefore, we get

$$
\left\|t^{\alpha_{0} \beta_{0}}|\nabla|^{\beta_{0}+s_{c}} \int_{0}^{\frac{1}{2} t} e^{i(t-s) \Delta} \chi_{\leq 1}(s) P_{M}\left(|v|^{p} v\right) d s\right\|_{l_{M}^{\infty} L_{t}^{2} L_{x}^{\frac{2 d}{d-2}}\left(\{M \geq 1\} \times\left\{|t| \geq M^{\left.\left.-\frac{1}{\alpha_{0}}\right\} \times \mathbb{R}^{d}\right)}\right.\right.} \lesssim\left\|v_{0}\right\|_{\dot{H}^{s_{c}\left(\mathbb{R}^{d}\right)^{2}}}^{p+1} .
$$

Estimates on (4.10c). By the Sobolev and the Bernstein inequalities, we have

$$
\begin{aligned}
\left\|t^{\alpha_{0} \beta_{0}}|\nabla|^{\beta_{0}+s_{c}} \int_{\frac{1}{2} t}^{t} e^{i(t-s) \Delta} \chi_{\leq 1}(s) P_{M}\left(|v|^{p} v\right) d s\right\|_{L_{t}^{2} L_{x}^{\frac{2 d}{d-2}}\left(\left\{|t| \geq M^{-\frac{1}{\alpha_{0}}}\right\} \times \mathbb{R}^{d}\right)} \\
\quad \lesssim M^{\beta_{0}+s_{c}}\left\|\int_{\frac{1}{2} t}^{t} e^{i(t-s) \Delta} \chi_{\leq 1}(s) s^{\alpha_{0} \beta_{0}} P_{M}\left(|v|^{p} v\right) d s\right\|_{L_{t}^{2} L_{x}^{\frac{2 d}{d-2}}\left(\mathbb{R} \times \mathbb{R}^{d}\right)} .
\end{aligned}
$$

Now we split it into two cases: $p \leq 1$ and $p>1$.

If $p \leq 1$, using Lemma 2.9 and (4.15), (4.10c) is further bounded by

$$
M^{\beta_{0}+s_{c}}\left\|\chi_{\leq 1}(t) t^{\alpha_{0} \beta_{0}} P_{M}\left(|v|^{p} v\right)\right\|_{L_{t}^{\frac{2}{p+1}} L_{x}^{r^{\prime}}\left(\mathbb{R} \times \mathbb{R}^{d}\right)},
$$

where $r_{5}$ is the parameter satisfying

$$
\frac{1}{r_{5}}=\frac{1}{2}-\frac{1-p}{d}
$$

In particular, this parameter verifies the following Hölder inequality,

$$
\left\||f|^{p} g\right\|_{L^{r_{5}^{\prime}}} \lesssim\|f\|_{L_{x}^{\frac{d p}{2-p}}\left(\mathbb{R}^{d}\right)}\|g\|_{L_{x}^{\frac{2 d}{d-2}}\left(\mathbb{R}^{d}\right)} .
$$

Now we consider the term

$$
\left\|P_{M}\left(|v|^{p} v\right)\right\|_{L_{x}^{r_{5}^{\prime}\left(\mathbb{R}^{d}\right)}}
$$


We write

$$
\begin{aligned}
\left\|P_{M}\left(|v|^{p} v\right)\right\|_{L_{x}^{r_{5}^{\prime}\left(\mathbb{R}^{d}\right)}} \leq & \left\|P_{M}\left(\left|P_{\leq M} v\right|^{p} P_{\leq M} v\right)\right\|_{L_{x}^{r_{5}^{\prime}}\left(\mathbb{R}^{d}\right)} \\
& +\left\|P_{M}\left(|v|^{p} v-\left|P_{\leq M} v\right|^{p} P_{\leq M} v\right)\right\|_{L_{x}^{r_{5}^{\prime}}\left(\mathbb{R}^{d}\right)} .
\end{aligned}
$$

We choose $s_{*}<0$ suitably close to 0 such that for any $s_{c} \in\left(s_{*}, 0\right)$,

$$
s_{c}+\beta_{0}>0 \text {. }
$$

Then for (4.18), by Bernstein's inequality, we have

$$
\begin{aligned}
& \left\|P_{M}\left(\left|P_{\leq M} v\right|^{p} P_{\leq M} v\right)\right\|_{L_{x}^{r_{5}^{\prime}\left(\mathbb{R}^{d}\right)}} \\
& \quad \lesssim M^{-\left(s_{c}+\beta_{0}+\epsilon\right)}\left\||\nabla|^{s_{c}+\beta_{0}+\epsilon} P_{M}\left(\left|P_{\leq M} v\right|^{p} P_{\leq M} v\right)\right\|_{L_{x}^{r_{5}^{\prime}\left(\mathbb{R}^{d}\right)}},
\end{aligned}
$$

where $\epsilon$ is a small positive constant such that $s_{c}+\beta_{0}+\epsilon<p+1$. Then by Lemma 2.2, we further obtain

$$
\begin{aligned}
& \left\|P_{M}\left(\left|P_{\leq M} v\right|^{p} P_{\leq M} v\right)\right\|_{L_{x}^{r^{\prime}\left(\mathbb{R}^{d}\right)}} \\
& \quad \lesssim M^{-\left(s_{c}+\beta_{0}+\epsilon\right)}\left\||\nabla|^{s_{c}+\beta_{0}+\epsilon} P_{\leq M} v\right\|_{L_{x}^{\frac{2 d}{d-2}\left(\mathbb{R}^{d}\right)}}\left\|P_{\leq M} v\right\|_{L_{x}^{\frac{d p}{2-p}}\left(\mathbb{R}^{d}\right)}^{p} .
\end{aligned}
$$

Now by Littlewood-Paley's decomposition, we write

$$
\left\||\nabla|^{s_{c}+\beta_{0}+\epsilon} P_{\leq M} v\right\|_{L_{x}^{\frac{2 d}{d-2}}\left(\mathbb{R}^{d}\right)} \lesssim\left\||\nabla|^{s_{c}+\beta_{0}+\epsilon} P_{\leq 1} v\right\|_{L_{x}^{\frac{2 d}{d-2}}\left(\mathbb{R}^{d}\right)}+\sum_{1 \leq M_{1} \leq M} M_{1}^{\epsilon}\left\||\nabla|^{s_{c}+\beta_{0}} P_{M_{1}} v\right\|_{L_{x}^{\frac{2 d}{d-2}}\left(\mathbb{R}^{d}\right)} .
$$

Note that by Lemma 4.3 ,

$$
\left\||\nabla|^{s_{c}+\beta_{0}+\epsilon} P_{\leq 1} v\right\|_{L_{t}^{\infty} L_{x}^{\frac{2 d}{d-2}}\left(\mathbb{R} \times \mathbb{R}^{d}\right)} \lesssim\left\||\nabla|^{s_{c}} P_{\leq 1} v\right\|_{L_{t}^{\infty} L_{x}^{2}\left(\mathbb{R} \times \mathbb{R}^{d}\right)} \lesssim\left\|v_{0}\right\|_{\dot{H}^{s_{c}}\left(\mathbb{R}^{d}\right)} .
$$

Hence, we obtain that

$$
\begin{aligned}
& M^{\beta_{0}+s_{c}}\left\|\chi_{\leq 1}(t) t^{\alpha_{0} \beta_{0}} P_{M}\left(\left|P_{\leq M} v\right|^{p} P_{\leq M} v\right)\right\|_{L_{t}^{\frac{2}{p+1}} L_{x}^{r_{5}^{\prime}}\left(\mathbb{R} \times \mathbb{R}^{d}\right)} \\
& \lesssim M^{-\epsilon} \| \chi_{\leq 1}(t) t^{\alpha_{0} \beta_{0}}\left(\left\|v_{0}\right\|_{\dot{H}^{s_{c}\left(\mathbb{R}^{d}\right)}}+\sum_{1 \leq M_{1} \leq M} M_{1}^{\epsilon}\left\||\nabla|^{s_{c}+\beta_{0}} P_{M_{1}} v\right\|_{L_{x}^{\frac{2 d}{d-2}}\left(\mathbb{R}^{d}\right)}\right) \\
& \cdot\left\|P_{\leq M} v\right\|_{L_{x}^{\frac{d p}{2-p}}\left(\mathbb{R}^{d}\right)}^{p}\|\|_{L_{t}^{\frac{2}{p+1}}(\mathbb{R})} \\
& \lesssim M^{-\epsilon}\left\|v_{0}\right\|_{\dot{H}^{s_{c}}\left(\mathbb{R}^{d}\right)}\|v\|_{L_{t}^{2} L_{x}^{\frac{d p}{2-p}}\left(\mathbb{R} \times \mathbb{R}^{d}\right)} \\
& +M^{-\epsilon}\left(\sum_{1 \leq M_{1} \leq M} M_{1}^{\epsilon}\left\|t^{\alpha_{0} \beta_{0}}|\nabla|^{s_{c}+\beta_{0}} P_{M_{1}} v\right\|_{L_{t}^{2} L_{x}^{\frac{2 d}{d-2}}\left(\mathbb{R} \times \mathbb{R}^{d}\right)}\right) \cdot\|v\|_{L_{t}^{2} L_{x}^{\frac{d p}{2-p}}\left(\mathbb{R} \times \mathbb{R}^{d}\right)} .
\end{aligned}
$$

Now by Lemma 4.3 and the definition of $X\left(\alpha_{0}, \beta_{0}\right)$, we have

$$
\|v\|_{L_{t}^{2} L_{x}^{\frac{d p}{2-p}}\left(\mathbb{R} \times \mathbb{R}^{d}\right)} \lesssim\left\|v_{0}\right\|_{\dot{H}^{s_{c}}\left(\mathbb{R}^{d}\right)},
$$

and

$$
\left\|t^{\alpha_{0} \beta_{0}}|\nabla|^{s_{c}+\beta_{0}} P_{M_{1}} v\right\|_{L_{t}^{2} L_{x}^{\frac{2 d}{d-2}}\left(\mathbb{R} \times \mathbb{R}^{d}\right)} \lesssim\|v\|_{X\left(\alpha_{0}, \beta_{0}\right)} .
$$

Inserting these two estimates into (4.20), then (4.20) is controlled by

$$
M^{-\epsilon}\left\|v_{0}\right\|_{\dot{H}^{s_{c}\left(\mathbb{R}^{d}\right)}}^{p+1}+M^{-\epsilon} \sum_{1 \leq M_{1} \leq M} M_{1}^{\epsilon}\|v\|_{X\left(\alpha_{0}, \beta_{0}\right)} \cdot\left\|v_{0}\right\|_{\dot{H}^{s_{c}}\left(\mathbb{R}^{d}\right)}^{p} .
$$


Taking summation, we obtain

$$
\begin{array}{r}
M^{\beta_{0}+s_{c}}\left\|\chi_{\leq 1}(t) t^{\alpha_{0} \beta_{0}} P_{M}\left(\left|P_{\leq M} v\right|^{p} P_{\leq M} v\right)\right\|_{L_{t}^{\frac{2}{p+1}} L_{x}^{r_{5}^{\prime}}\left(\mathbb{R} \times \mathbb{R}^{d}\right)} \\
\lesssim\left\|v_{0}\right\|_{\dot{H}^{s_{c}}\left(\mathbb{R}^{d}\right)}^{p+1}+\left\|v_{0}\right\|_{\dot{H}^{s_{c}}\left(\mathbb{R}^{d}\right)}^{p}\|v\|_{X\left(\alpha_{0}, \beta_{0}\right)}
\end{array}
$$

For (4.19), by Bernstein's inequality, we have

$$
\begin{aligned}
\| P_{M}\left(|v|^{p} v\right. & \left.-\left|P_{\leq M} v\right|^{p} P_{\leq M} v\right) \|_{L_{x}^{r^{\prime}}\left(\mathbb{R}^{d}\right)} \\
& \lesssim\left\|P_{\geq M} v\right\|_{L_{x}^{\frac{2 d}{d-2}}\left(\mathbb{R}^{d}\right)}\|v\|_{L_{x}^{2-p}\left(\mathbb{R}^{d}\right)}^{p} \\
& \lesssim \sum_{M_{1} \geq M}\left\|P_{M_{1}} v\right\|_{L_{x}^{\frac{2 d}{d-2}}\left(\mathbb{R}^{d}\right)}\|v\|_{L_{x}^{\frac{d p}{2-p}}\left(\mathbb{R}^{d}\right)}^{p} \\
& \lesssim \sum_{M_{1} \geq M} M_{1}^{-\left(s_{c}+\beta_{0}\right)}\left\||\nabla|^{s_{c}+\beta_{0}} P_{M_{1}} v\right\|_{L_{x}^{\frac{2 d}{d-2}}\left(\mathbb{R}^{d}\right)}\|v\|_{L_{x}^{2-p}\left(\mathbb{R}^{d}\right)}^{p} .
\end{aligned}
$$

Hence, we obtain that

$$
\begin{aligned}
M^{\beta_{0}+s_{c}} & \left\|\chi_{\leq 1}(t) t^{\alpha_{0} \beta_{0}} P_{M}\left(|v|^{p} v-\left|P_{\leq M} v\right|^{p} P_{\leq M} v\right)\right\|_{L_{t}^{\frac{2}{p+1}} L_{x}^{r_{5}^{\prime}}\left(\mathbb{R} \times \mathbb{R}^{d}\right)} \\
& \lesssim M^{\beta_{0}+s_{c}} \sum_{M_{1} \geq M} M_{1}^{-\left(s_{c}+\beta_{0}\right)}\left\|\chi_{\leq 1}(t) t^{\alpha_{0} \beta_{0}}\right\||\nabla|^{s_{c}+\beta_{0}} P_{M_{1}} v\left\|_{L_{x}^{\frac{2 d}{d-2}}\left(\mathbb{R}^{d}\right)}\right\| v\left\|_{L_{x}^{\frac{d p}{2-p}}\left(\mathbb{R}^{d}\right)}^{p}\right\| \|_{L_{t}^{\frac{2}{p+1}}(\mathbb{R})} \\
& \lesssim M^{\beta_{0}+s_{c}} \sum_{M_{1} \geq M} M_{1}^{-\left(s_{c}+\beta_{0}\right)}\left\|t^{\alpha_{0} \beta_{0}}|\nabla|^{s_{c}+\beta_{0}} P_{M_{1}} v\right\|_{L_{t}^{2} L_{x}^{\frac{2 d}{d-2}}\left(\mathbb{R} \times \mathbb{R}^{d}\right)}\|v\|_{L_{t}^{2} L_{x}^{\frac{d p}{2-p}}\left(\mathbb{R} \times \mathbb{R}^{d}\right)}^{p} .
\end{aligned}
$$

Similar as above, it is further bounded by

$$
M^{\beta_{0}+s_{c}} \sum_{M_{1} \geq M} M_{1}^{-\left(s_{c}+\beta_{0}\right)}\|v\|_{X\left(\alpha_{0}, \beta_{0}\right)} \cdot\left\|v_{0}\right\|_{\dot{H}^{s_{c}\left(\mathbb{R}^{d}\right)}}^{p} .
$$

Taking summation, we obtain that

$$
\begin{gathered}
M^{\beta_{0}+s_{c}}\left\|\chi_{\leq 1}(t) t^{\alpha_{0} \beta_{0}} P_{M}\left(|v|^{p} v-\left|P_{\leq M} v\right|^{p} P_{\leq M} v\right)\right\|_{L_{t}^{p+1} L_{x}^{r^{\prime}}\left(\mathbb{R} \times \mathbb{R}^{d}\right)} \\
\lesssim\left\|v_{0}\right\|_{\dot{H}^{s_{c}}\left(\mathbb{R}^{d}\right)}^{p}\|v\|_{X\left(\alpha_{0}, \beta_{0}\right)} .
\end{gathered}
$$

Now, together with (4.16), (4.18), (4.19), (4.21) and (4.22), we obtain the estimates on (4.10c) in the case of $p \leq 1$ as

$$
\begin{gathered}
\left\|t^{\alpha_{0} \beta_{0}}|\nabla|^{\beta_{0}+s_{c}} \int_{\frac{1}{2} t}^{t} e^{i(t-s) \Delta} \chi_{\leq 1}(s) P_{M}\left(|v|^{p} v\right) d s\right\|_{L_{t}^{2} L_{x}^{\frac{2 d}{d-2}}\left(\left\{|t| \geq M^{-\frac{1}{\alpha_{0}}}\right\} \times \mathbb{R}^{d}\right)} \\
\lesssim\left\|v_{0}\right\|_{\dot{H}^{s^{c}\left(\mathbb{R}^{d}\right)}}^{p+1}+\left\|v_{0}\right\|_{\dot{H}^{s_{c}}\left(\mathbb{R}^{d}\right)}^{p}\|v\|_{X\left(\alpha_{0}, \beta_{0}\right)} .
\end{gathered}
$$

Next, we consider the case when $p>1$ (now $d=2,3$ ), which can be treated similarly as above. Then using Lemma 2.9] and (4.15), (4.10c) is bounded by

$$
M^{\beta_{0}+s_{c}}\left\|\chi_{\leq 1}(t) t^{\alpha_{0} \beta_{0}} P_{M}\left(|v|^{p} v\right)\right\|_{L_{t}^{1} L_{x}^{2}\left(\mathbb{R} \times \mathbb{R}^{d}\right)} .
$$

Arguing similarly as the case of $p \leq 1$, and based on the Hölder inequality,

$$
\left\||f|^{p} f\right\|_{L_{t}^{1} L_{x}^{2}\left(\mathbb{R} \times \mathbb{R}^{d}\right)} \leq\|f\|_{L_{t}^{2} L_{x}^{\frac{2 d}{d-2}}\left(\mathbb{R} \times \mathbb{R}^{d}\right)}\|f\|_{L_{t}^{2 p} L_{x}^{d p}\left(\mathbb{R} \times \mathbb{R}^{d}\right)}^{p}
$$


we also obtain (4.23) when $p>1$.

Now collecting the three estimates on (4.10), we get that

$$
(4.8) \lesssim\left\|v_{0}\right\|_{\dot{H}^{s_{c}\left(\mathbb{R}^{d}\right)}}+\left\|v_{0}\right\|_{\dot{H}^{s\left(\mathbb{R}^{d}\right)}}^{p+1}+\left\|v_{0}\right\|_{\dot{H}^{s^{c}\left(\mathbb{R}^{d}\right)}}^{p}\|v\|_{X\left(\alpha_{0}, \beta_{0}\right)} .
$$

Combining this estimate with (4.9), we obtain that

$$
\|v\|_{X\left(\alpha_{0}, \beta_{0}\right)} \lesssim\left\|v_{0}\right\|_{\dot{H}^{s_{c}\left(\mathbb{R}^{d}\right)}}+\left\|v_{0}\right\|_{\dot{H}^{s_{c}\left(\mathbb{R}^{d}\right)}}^{p+1}+\left\|v_{0}\right\|_{\dot{H}^{s_{c}\left(\mathbb{R}^{d}\right)}}^{p}\|v\|_{X\left(\alpha_{0}, \beta_{0}\right)} .
$$

Using (4.4) and choosing $\delta_{0}$ suitably small, we give the proof of the proposition.

As a consequence, we have

Corollary 4.5. There exists $s_{*}<0$, such that for any $s_{c} \in\left[s_{*}, 0\right)$, the following result holds.

$$
\left\|\chi_{\geq 1}(t) v\right\|_{L_{t}^{2} L_{x}^{\frac{2 d}{d-2}}\left([0,2] \times \mathbb{R}^{d}\right)} \lesssim\left\|v_{0}\right\|_{\dot{H}^{s_{c}}\left(\mathbb{R}^{d}\right)} .
$$

Proof. For the low frequency part, by Lemma 4.3 we have

$$
\left\|P_{\leq 1} v\right\|_{L_{t}^{2} L_{x}^{\frac{2 d}{d-2}}\left([0,2] \times \mathbb{R}^{d}\right)} \lesssim\left\|P_{\leq 1}|\nabla|^{s_{c}} v\right\|_{L_{t}^{2} L_{x}^{\frac{2 d}{d-2}}\left([0,2] \times \mathbb{R}^{d}\right)} \lesssim\left\|v_{0}\right\|_{\dot{H}^{s_{c}}\left(\mathbb{R}^{d}\right)} .
$$

For the high frequency part, from Proposition 4.4 and Sobolev's inequality, we have that for any $M \geq 1$,

$$
\left\|t^{\alpha_{1} \beta_{0}}|\nabla|^{\beta_{0}+s_{c}} P_{M} v\right\|_{L_{t}^{2} L_{x}^{\frac{2 d}{d-2}}\left(\left[\frac{1}{2}, 2\right] \times \mathbb{R}^{d}\right)} \lesssim\left\|v_{0}\right\|_{\dot{H}^{s_{c}}\left(\mathbb{R}^{d}\right)} .
$$

This implies that

$$
\left\|P_{M} v\right\|_{L_{t}^{2} L_{x}^{\frac{2 d}{d-2}}\left(\left[\frac{1}{2}, 2\right] \times \mathbb{R}^{d}\right)} \lesssim M^{-\left(\beta_{0}+s_{c}\right)}\left\|v_{0}\right\|_{\dot{H}^{s_{c}}\left(\mathbb{R}^{d}\right)} .
$$

Choosing $s_{*}$ small enough such that $\beta_{0}+s_{c}>0$, then taking summation on $M$, we have that

$$
\left\|P_{\geq 1} v\right\|_{L_{t}^{2} L_{x}^{\frac{2 d}{d-2}}\left(\left[\frac{1}{2}, 2\right] \times \mathbb{R}^{d}\right)} \lesssim\left\|v_{0}\right\|_{\dot{H}^{s_{c}}\left(\mathbb{R}^{d}\right)} .
$$

Hence, we obtain the desired estimates.

Furthermore, we also need the following long time estimate on $v$.

Proposition 4.6. There exists $s_{*}<0$, such that the following properties hold. For any $t \geq 2, s_{c} \in\left[s_{*}, 0\right]$ and any $r$ such that

$$
\frac{1-p}{2}+\frac{d-1}{p+1}\left|s_{*}\right|<\frac{1}{r} \leq \frac{1-p}{2}+\frac{p+1}{d}-\frac{(p+1)\left|s_{*}\right|}{d},
$$

then

$$
\|v(t)\|_{L_{x}^{r}\left(\mathbb{R}^{d}\right)} \lesssim t^{-(d-1)\left(\frac{1}{2}-\frac{1}{r}\right)} .
$$

Moreover,

$$
\|v\|_{L_{t}^{1} L_{x}^{\frac{2}{1-p}}\left([2, \infty) \times \mathbb{R}^{d}\right)} \lesssim 1
$$


Proof. First, we consider (4.26). By the Duhamel formula, we have

$$
\|v\|_{L_{x}^{r}\left(\mathbb{R}^{d}\right)} \lesssim\left\|e^{i t \Delta} v_{0}\right\|_{L_{x}^{r}\left(\mathbb{R}^{d}\right)}+\int_{0}^{t}\left\|e^{i(t-s) \Delta} \chi_{\leq 1}(s)|u|^{p} u\right\|_{L_{x}^{r}\left(\mathbb{R}^{d}\right)} d s .
$$

From Proposition 3.1 and (4.4), we get that for $r>2$,

$$
\left\|e^{i t \Delta} v_{0}\right\|_{L_{x}^{r}\left(\mathbb{R}^{d}\right)} \lesssim N^{-(d-2)\left(\frac{1}{2}-\frac{1}{r}\right)-s_{c}}|t|^{-(d-1)\left(\frac{1}{2}-\frac{1}{r}\right)}\left\|P_{\geq N} u_{0}\right\|_{\dot{H}^{s_{c}}\left(\mathbb{R}^{d}\right)} \lesssim \delta_{0}|t|^{-(d-1)\left(\frac{1}{2}-\frac{1}{r}\right)} .
$$

Here we shall choose $\left|s^{*}\right|$ suitably small such that $-(d-2)\left(\frac{1}{2}-\frac{1}{r}\right)+\left|s_{*}\right| \leq 0$.

Note that when $t \geq 2, s \leq \frac{11}{10}$, we have that $|t-s| \sim t$. Then from Lemma 2.8, we have

$$
\begin{aligned}
\int_{0}^{t}\left\|e^{i(t-s) \Delta} \chi_{\leq 1}(s)|u|^{p} u\right\|_{L_{x}^{r}\left(\mathbb{R}^{d}\right)} d s \\
\quad \lesssim \int_{0}^{t}|t-s|^{-d\left(\frac{1}{2}-\frac{1}{r}\right)} \chi_{\leq 1}(s)\left\||u|^{p} u\right\|_{L_{x}^{r^{\prime}\left(\mathbb{R}^{d}\right)}} d s \\
\lesssim|t|^{-d\left(\frac{1}{2}-\frac{1}{r}\right)} \int_{0}^{2}\|u(s)\|_{L_{x}^{r^{\prime}(p+1)}\left(\mathbb{R}^{d}\right)}^{p+1} d s .
\end{aligned}
$$

Setting $q$ such that

$$
q \geq 2, \quad \frac{2}{q}+\frac{d}{r^{\prime}(p+1)}=\frac{d}{2}-s_{c} .
$$

Note that the condition of (4.25) in the right-hand side assures that $q \geq 2$. Moreover, when $r$ satisfies that $\frac{1}{r}>\frac{1-p}{2}+\frac{d-1}{p+1}\left|s_{*}\right|$, then $\left(q, r^{\prime}(p+1),-s_{c}\right)$ verifies (2.5) and $q \geq p+1$ (see Remark 2.12). Hence, by Hölder's inequality and Lemma 4.1, we have

$$
\int_{0}^{2}\|u\|_{L_{x}^{r^{\prime}(p+1)}\left(\mathbb{R}^{d}\right)}^{p+1} d s \lesssim\|u\|_{L_{t}^{q} L_{x}^{r^{\prime}(p+1)}\left([0,2] \times \mathbb{R}^{d}\right)}^{p+1} \lesssim 1
$$

Hence, we obtain that

$$
\int_{0}^{t}\left\|e^{i(t-s) \Delta} \chi_{\leq 1}(s)|u|^{p} u\right\|_{L_{x}^{r}\left(\mathbb{R}^{d}\right)} d s \lesssim|t|^{-d\left(\frac{1}{2}-\frac{1}{r}\right)} .
$$

This last estimate combined with (4.28), gives (4.26).

Now we consider (4.27). By the Duhamel formula, we have

$$
\begin{aligned}
\|v\|_{L_{t}^{1} L_{x}^{\frac{2}{1-p}}\left([2, \infty) \times \mathbb{R}^{d}\right)} \lesssim & \left\|e^{i t \Delta} v_{0}\right\|_{L_{t}^{1} L_{x}^{\frac{2}{1-p}}\left([2, \infty) \times \mathbb{R}^{d}\right)} \\
& +\left\|\int_{0}^{t} e^{i(t-s) \Delta} \chi_{\leq 1}(s) P_{\leq 1}\left(|u|^{p} u\right) d s\right\|_{L_{t}^{1} L_{x}^{1-p}\left([2, \infty) \times \mathbb{R}^{d}\right)} \\
& +\left\|\int_{0}^{t} e^{i(t-s) \Delta} \chi_{\leq 1}(s) P_{\geq 1}\left(|u|^{p} u\right) d s\right\|_{L_{t}^{1} L_{x}^{\frac{2}{1-p}}\left([2, \infty) \times \mathbb{R}^{d}\right)} .
\end{aligned}
$$

For the term (4.29a), it follows by (4.28) that

$$
\left\|e^{i t \Delta} v_{0}\right\|_{L_{t}^{1} L_{x}^{1-p}\left([2, \infty) \times \mathbb{R}^{d}\right)} \lesssim \delta_{0}\left\||t|^{-(d-1) \frac{p}{2}}\right\|_{L_{t}^{1}([2, \infty))} \lesssim 1 .
$$


Here we shall choose $\left|s^{*}\right|$ suitably small such that $(d-1) p>2$. For the term (4.29b), by Bernstein's inequality, we have that

$$
\begin{aligned}
\| \int_{0}^{t} e^{i(t-s) \Delta} & \chi_{\leq 1}(s) P_{\leq 1}\left(|u|^{p} u\right) d s \|_{L_{t}^{1} L_{x}^{1-p}\left([2, \infty) \times \mathbb{R}^{d}\right)} \\
& \lesssim\left\|\int_{0}^{t} e^{i(t-s) \Delta} \chi_{\leq 1}(s)|u|^{p} u d s\right\|_{L_{t}^{1} L_{x}^{\left(\frac{r_{6}}{1+p}\right)^{\prime}}{ }_{\left([2, \infty) \times \mathbb{R}^{d}\right)}} \\
& \lesssim\left\|\int_{0}^{2}|t-s|^{-d\left(\frac{p}{2}-a_{0}(1+p)\right)}\right\||u|^{p} u\left\|_{L_{x}^{\frac{r_{6}}{1+p}}\left(\mathbb{R}^{d}\right)} d s\right\|_{L_{t}^{1}([2, \infty)} \lesssim\|u\|_{L_{t}^{q_{6}} L_{x}^{r_{6}}\left([0,2] \times \mathbb{R}^{d}\right)^{1}}, \\
& \lesssim\left\||t|^{-d\left(\frac{p}{2}-a_{0}(1+p)\right)}\right\|_{L_{t}^{1}([2, \infty)}\left\||u|^{p} u\right\|_{L_{t}^{1} L_{x}^{1+p}\left([0,2] \times \mathbb{R}^{d}\right)} \lesssim
\end{aligned}
$$

where $a_{0}, r_{6}, q_{6}$ verisfy

$$
a_{0}=\frac{\left|s_{*}\right|}{d-2} ; \quad \frac{1}{r_{6}}=\frac{1}{2}-a_{0} ; \quad \frac{1}{q_{6}}=\frac{1}{2}\left(a_{0} d-s_{c}\right) .
$$

Note that the triple $\left(q_{6}, r_{6},-s_{c}\right)$ verifies (2.5), then by Lemma 4.1, we have that

$$
\left\|\int_{0}^{t} e^{i(t-s) \Delta} \chi_{\leq 1}(s) P_{\leq 1}\left(|u|^{p} u\right) d s\right\|_{L_{t}^{1} L_{x}^{\frac{2}{1-p}}\left([2, \infty) \times \mathbb{R}^{d}\right)} \lesssim 1 .
$$

For the term (4.29c), applying (2.10) (noting that in our case, $\theta=\frac{d}{3 d-2}-$ when $s_{c}=0$, thus (2.9) verifies when $\left|s_{*}\right|$ is suitable small) to give that

$$
\left\|\int_{0}^{t} e^{i(t-s) \Delta} \chi_{\leq 1}(s) P_{\geq 1}\left(|u|^{p} u\right) d s\right\|_{L_{t}^{1} L_{x}^{\frac{2}{1-p}}\left([2, \infty) \times \mathbb{R}^{d}\right)} \lesssim\left\||\nabla|^{\tilde{\gamma}} P_{\geq 1}\left(|u|^{p} u\right)\right\|_{L_{t}^{\tilde{q}} L_{x}^{\tilde{r}}\left([0,2] \times \mathbb{R}^{d}\right)^{.}}
$$

Here we choose the parameters $\tilde{\gamma}, \tilde{q}, \tilde{r}$ such that

$\frac{1}{\tilde{r}}=\frac{p}{r_{6}}+\frac{1}{r_{0}} ; \quad \frac{1}{\tilde{q}}=\frac{p}{q_{6}}+\frac{1}{2} ; \quad \tilde{\gamma}=\left(\frac{1}{2}-\frac{1}{r_{0}}+a_{0} p\right)\left(\frac{1}{r_{0}}-\frac{1}{2}+\frac{p}{2}\right)^{-1}\left(\frac{d-2}{2}-\gamma_{0}-\frac{(1-p) d}{2}\right)$.

Note that when $\left|s_{*}\right|$ is suitably small, then $\tilde{\gamma} \leq \gamma_{0}+s_{c}$ (indeed, when $s_{c}=0$ then $\tilde{\gamma}=$ $\left.\frac{d^{2}}{(2 d-1)(3 d-2)}+<\gamma_{0}\right)$. Hence, by Bernstein's and Hölder's inequalities, and Lemma 2.2 .

$$
\begin{aligned}
\left\||\nabla|^{\tilde{\gamma}} P_{\geq 1}\left(|u|^{p} u\right)\right\|_{L_{t}^{\tilde{q}} L_{x}^{\tilde{r}}\left([0,2] \times \mathbb{R}^{d}\right)} & \lesssim\left\||\nabla|^{\gamma_{0}+s_{c}}\left(|u|^{p} u\right)\right\|_{L_{t}^{\tilde{q}} L_{x}^{\tilde{r}}\left([0,2] \times \mathbb{R}^{d}\right)} \\
& \lesssim\left\||\nabla|^{\gamma_{0}+s_{c}} u\right\|_{L_{t}^{2} L_{x}^{r_{0}}([0,2] \times \mathbb{R})}\|u\|_{L_{t}^{q_{6}} L_{x}^{r_{6}}\left([0,2] \times \mathbb{R}^{d}\right)}^{p} .
\end{aligned}
$$

Therefore, by Lemma 4.1, we get that

$$
\left\||\nabla|^{\tilde{\gamma}} P_{\geq 1}\left(|u|^{p} u\right)\right\|_{L_{t}^{\tilde{q}} L_{x}^{\tilde{r}}\left([0,2] \times \mathbb{R}^{d}\right)} \lesssim 1
$$

This implies that

$$
\left\|\int_{0}^{t} e^{i(t-s) \Delta} \chi_{\leq 1}(s) P_{\geq 1}\left(|u|^{p} u\right) d s\right\|_{L_{t}^{1} L_{x}^{\frac{1}{1-p}}\left([2, \infty) \times \mathbb{R}^{d}\right)} \lesssim 1 .
$$

Collecting the three estimates on (4.29), we obtain (4.27).

\section{The Proof of Theorem 1.1}

In this section, we prove Theorem 1.1 . 
5.1. Nonlinear estimates on $w$. In this subsection, we give some nonlinear estimates of the solution with the low frequency initial data.

First, for $u_{0} \in \dot{H}^{s_{c}}\left(\mathbb{R}^{d}\right), s_{c}<0$ with supp $u_{0} \subset\{x:|x| \leq 1\}$, we claim that

$$
w_{0} \in L^{2}\left(\mathbb{R}^{d}\right) \quad \text { and } \quad\left\|w_{0}\right\|_{L^{2}\left(\mathbb{R}^{d}\right)} \lesssim N^{-s_{c}}\left\|u_{0}\right\|_{\dot{H}^{s^{c}\left(\mathbb{R}^{d}\right)}} .
$$

Indeed, by the mismatch estimate in Lemma 2.5,

$$
\left\|\chi_{\geq 10}\left(P_{\geq N} u_{0}\right)\right\|_{L^{2}\left(\mathbb{R}^{d}\right)} \lesssim N^{-10}\left\|u_{0}\right\|_{\dot{H}^{s_{c}}\left(\mathbb{R}^{d}\right)},
$$

and by the Bernstein estimate,

$$
\left\|P_{\leq N} u_{0}\right\|_{L^{2}\left(\mathbb{R}^{d}\right)} \lesssim N^{-s_{c}}\left\|u_{0}\right\|_{\dot{H}^{s_{c}}\left(\mathbb{R}^{d}\right)} .
$$

This gives (5.1).

To clear our argument, in the following we only consider the case of $p<1$, which is assured by $d \geq 4$.

The first we need is the following local estimates of $w$ in more regular spaces.

Lemma 5.1. The Cauchy problem (4.6) is locally well-posed in $L^{2}\left(\mathbb{R}^{d}\right)$ in the time interval $[0,2]$. In particular, the solution $w$ satisfies that

$$
\|w\|_{L_{t}^{2} L_{x}^{\frac{2 d}{d-2}}\left([0,2] \times \mathbb{R}^{d}\right)}+\|w\|_{L_{t}^{\infty} L_{x}^{2}\left([0,2] \times \mathbb{R}^{d}\right)} \lesssim 1+\left\|w_{0}\right\|_{L^{2}\left(\mathbb{R}^{d}\right)} .
$$

Proof. By Lemma 2.9, we have

$$
\|w\|_{L_{t}^{2} L_{x}^{\frac{2 d}{d-2}\left([0,2] \times \mathbb{R}^{d}\right)}}+\|w\|_{L_{t}^{\infty} L_{x}^{2}\left([0,2] \times \mathbb{R}^{d}\right)} \lesssim\left\|w_{0}\right\|_{L^{2}\left(\mathbb{R}^{d}\right)}+\left\||u|^{p} u-\chi_{\leq 1}(t)|v|^{p} v\right\|_{L_{t}^{\frac{2}{p+1}} L_{x}^{r_{5}^{\prime}\left([0,2] \times \mathbb{R}^{d}\right)}} .
$$

Next, we consider

Note that

$$
\left\||u|^{p} u-\chi_{\leq 1}(t)|v|^{p} v\right\|_{L_{t}^{\frac{2}{p+1}} L_{x}^{r_{5}^{\prime}}\left([0,2] \times \mathbb{R}^{d}\right)} .
$$

$$
\left.|| u\right|^{p} u-\chi_{\leq 1}(t)|v|^{p} v \mid \lesssim\left(|u|^{p}+\left|\tilde{\chi}_{\leq 1}(t) v\right|^{p}\right)\left(|w|+\left|\tilde{\chi}_{\geq 1}(t) v\right|\right) .
$$

Here we denote the time-dependent functions $\tilde{\chi}_{\leq 1}(t)=\chi_{\leq 1}^{\frac{1}{p+1}}(t)$ and $\tilde{\chi}_{\geq 1}(t)=1-\tilde{\chi}_{\leq 1}(t)$. Hence, by (4.17), we have that

$$
\begin{aligned}
& \left\||u|^{p} u-\chi \leq 1(t)|v|^{p} v\right\|_{L_{t}^{\frac{2}{p+1}} L_{x}^{r^{\prime}}\left([0,2] \times \mathbb{R}^{d}\right)} \\
& \lesssim\left(\|u\|_{L_{t}^{2} L_{x}^{\frac{d p}{2-p}}\left([0,2] \times \mathbb{R}^{d}\right)}^{p}+\|v\|_{L_{t}^{2} L_{x}^{\frac{d p}{2-p}}\left([0,2] \times \mathbb{R}^{d}\right)}^{p}\right) \\
& \cdot\left(\|w\|_{L_{t}^{2} L_{x}^{\frac{2 d}{d-2}}\left([0,2] \times \mathbb{R}^{d}\right)}+\left\|\chi_{\geq 1}(t) v\right\|_{L_{t}^{2} L_{x}^{\frac{2 d}{d-2}}\left([0,2] \times \mathbb{R}^{d}\right)}\right) .
\end{aligned}
$$

From Lemmas 4.1 and 4.3 , we have that

$$
\|u\|_{L_{t}^{2} L_{x}^{\frac{d p}{2-p}}\left([0,2] \times \mathbb{R}^{d}\right)} \lesssim \delta_{0} ; \quad\|v\|_{L_{t}^{2} L_{x}^{2-p}\left([0,2] \times \mathbb{R}^{d}\right)} \lesssim \delta_{0}
$$

Moreover, from Corollary 4.5, we have that

$$
\left\|\chi_{\geq 1}(t) v\right\|_{L_{t}^{2} L_{x}^{\frac{2 d}{d-2}}\left([0,2] \times \mathbb{R}^{d}\right)} \lesssim \delta_{0} .
$$


Hence, combining these last two estimates above and (5.2), we obtain

$$
\left\||u|^{p} u-\chi_{\leq 1}(t)|v|^{p} v\right\|_{L_{t}^{\frac{2}{p+1}} L_{x}^{r_{5}^{\prime}\left([0,2] \times \mathbb{R}^{d}\right)}} \lesssim \delta_{0}^{p}\left(\|w\|_{L_{t}^{2} L_{x}^{\frac{2 d}{d-2}}\left([0,2] \times \mathbb{R}^{d}\right)}+\delta_{0}\right) .
$$

Therefore, we obtain that

$$
\|w\|_{L_{t}^{2} L_{x}^{\frac{2 d}{d-2}}\left([0,2] \times \mathbb{R}^{d}\right)}+\|w\|_{L_{t}^{\infty} L_{x}^{2}\left([0,2] \times \mathbb{R}^{d}\right)} \lesssim 1+\left\|w_{0}\right\|_{L^{2}\left(\mathbb{R}^{d}\right)}+\delta_{0}^{p}\|w\|_{L_{t}^{2} L_{x}^{\frac{2 d}{d-2}}\left([0,2] \times \mathbb{R}^{d}\right)} .
$$

Choosing $\delta_{0}$ suitably small, we obtain

$$
\|w\|_{L_{t}^{2} L_{x}^{\frac{2 d}{d-2}}\left([0,2] \times \mathbb{R}^{d}\right)}+\|w\|_{L_{t}^{\infty} L_{x}^{2}\left([0,2] \times \mathbb{R}^{d}\right)} \lesssim 1+\left\|w_{0}\right\|_{L^{2}\left(\mathbb{R}^{d}\right)} .
$$

This finishes the proof of the lemma.

Next, we give the global estimates of $w$. The following is a modified mass estimate for $\dot{H}^{s_{c}}\left(\mathbb{R}^{d}\right)$-datum.

Proposition 5.2. Let $u_{0} \in \dot{H}^{s_{c}}\left(\mathbb{R}^{d}\right)$ and I be the lifespan of the solution $u$, then there exists $s_{*}<0$, such that for any $s_{c} \in\left(s_{*}, 0\right)$, the following estimate holds,

$$
\|w\|_{L_{t}^{\infty} L_{x}^{2}\left(I \times \mathbb{R}^{d}\right)}^{2} \lesssim N^{-2 s_{c}}\left\|u_{0}\right\|_{\dot{H}^{s_{c}}\left(\mathbb{R}^{d}\right)^{2}}^{2} .
$$

Proof. For simplicity, we denote $I=[0, T)$ and any $t \in I$,

$$
F(v, w)=|u|^{p} u-\chi_{\leq 1}(t)|v|^{p} v .
$$

Then from the equation (4.6), we have

$$
\partial_{t}\|w\|_{L_{x}^{2}}^{2}=2 \operatorname{Im} \int F(v, w) \bar{w} d x .
$$

We may assume $t \geq 2$, otherwise, the estimate has been included in Lemma 5.1. Then for any $t \in I, t \geq 2$, integrating in time from 2 to $t$, we obtain that

$$
\|w(t)\|_{L_{x}^{2}}^{2}=\|w(2)\|_{L^{2}}^{2}+2 \operatorname{Im} \int_{2}^{t} \int_{\mathbb{R}^{d}} F(v, w) \bar{w} d x d s .
$$

Now we consider

$$
2 \operatorname{Im} \int_{2}^{t} \int_{\mathbb{R}^{d}} F(v, w) \bar{w} d x d s
$$

To do this, we write

$$
F(v, w)=|u|^{p} w+|u|^{p} v-\chi_{\leq 1}(t)|v|^{p} v .
$$

Note that

$$
2 \operatorname{Im} \int_{2}^{t} \int_{\mathbb{R}^{d}}|u|^{p} w \bar{w} d x d s=0
$$

Hence, due to the time support, we have that

$$
2 \operatorname{Im} \int_{2}^{t} \int_{\mathbb{R}^{d}} F(v, w) \bar{w} d x d s=2 \operatorname{Im} \int_{2}^{t} \int_{\mathbb{R}^{d}}|u|^{p} v \bar{w} d x d s .
$$

Since $|u|^{p} \lesssim|w|^{p}+|v|^{p}$, this yields that

$$
\left|2 \operatorname{Im} \int_{2}^{t} \int_{\mathbb{R}^{d}} F(v, w) \bar{w} d x d s\right| \lesssim \int_{2}^{T} \int_{\mathbb{R}^{d}}|w|^{p+1}|v| d x d s+\int_{2}^{T} \int_{\mathbb{R}^{d}}|w||v|^{p+1} d x d s .
$$


For the first term in (5.4), we have that

$$
\int_{2}^{T} \int_{\mathbb{R}^{d}}|w|^{p+1}|v| d x d s \lesssim\|w\|_{L_{t}^{\infty} L_{x}^{2}\left([2, T) \times \mathbb{R}^{d}\right)}^{p+1}\|v\|_{L_{t}^{1} L_{x}^{\frac{2}{1-p}}\left([2, T) \times \mathbb{R}^{d}\right)} .
$$

Thus by Lemmas 4.6, we further get

$$
\int_{2}^{T} \int_{\mathbb{R}^{d}}|w|^{p+1}|v| d x d s \lesssim\|w\|_{L_{t}^{\infty} L_{x}^{2}\left([2, T) \times \mathbb{R}^{d}\right)}^{p+1} .
$$

For the second term in (5.4), we have

$$
\int_{2}^{T} \int_{\mathbb{R}^{d}}\left|w\left\|\left.v\right|^{p+1} d x d s \lesssim \int_{2}^{T}\right\| v(s)\left\|_{L_{x}^{2(p+1)}\left(\mathbb{R}^{d}\right)}^{p+1}\right\| w(s) \|_{L_{x}^{2}\left(\mathbb{R}^{d}\right)} d s .\right.
$$

Note that $2(p+1)$ satisfies (4.25) and $(d-1) p>2$ when $\left|s_{*}\right|$ suitably small, we further get

$$
\begin{aligned}
\int_{2}^{T} \int_{\mathbb{R}^{d}}|w \| v|^{p+1} d x d s & \lesssim \int_{2}^{T} s \frac{-(d-1) p}{2}\|w(s)\|_{L_{x}^{2}\left(\mathbb{R}^{d}\right)} d s \\
& \lesssim\|w\|_{L_{t}^{\infty} L_{x}^{2}\left([0, T) \times \mathbb{R}^{d}\right) .}
\end{aligned}
$$

Inserting (5.5) and (5.6) into (5.4), and then (5.3), we get that for any $t \in[2, T)$,

$$
\|w(t)\|_{L_{x}^{2}}^{2} \lesssim\|w(2)\|_{L^{2}}^{2}+\|w\|_{L_{t}^{\infty} L_{x}^{2}\left([0, T) \times \mathbb{R}^{d}\right)}+\|w\|_{L_{t}^{\infty} L_{x}^{2}\left([0, T) \times \mathbb{R}^{d}\right)}^{p+1} .
$$

This together with the result in Lemma 5.1 implies that

$$
\|w\|_{L_{t}^{\infty} L_{x}^{2}\left([0, T) \times \mathbb{R}^{d}\right)}^{2} \lesssim 1+\left\|w_{0}\right\|_{L^{2}}^{2}+\|w\|_{L_{t}^{\infty} L_{x}^{2}\left([0, T) \times \mathbb{R}^{d}\right)}+\|w\|_{L_{t}^{\infty} L_{x}^{2}\left([0, T) \times \mathbb{R}^{d}\right)}^{p+1} .
$$

Since $p<1$, by Cauchy-Schwartz's inequality, we obtain that

$$
\|w\|_{L_{t}^{\infty} L_{x}^{2}\left([0, T) \times \mathbb{R}^{d}\right)}^{2} \lesssim 1+\left\|w_{0}\right\|_{L^{2}}^{2} .
$$

This combining with (5.1) finishes the proof of the proposition.

5.2. Global existence. Now we prove $I=\mathbb{R}$. We only consider the positive time, the negative time being obtained in the same way. By the global result of $v$ obtained in Lemma 4.3, we only need to consider the global existence of $w$. It follows from the standard bootstrap argument and we only give its sketch. Fixing any $2 \leq t_{0} \in I$ and $0<\delta<1$, and treating similarly as in the proof of Lemma 5.1, we have that

$$
\begin{aligned}
\|w\|_{L_{t}^{2} L_{x}^{\frac{2 d}{d-2}}\left(\left[t_{0}, t_{0}+\delta\right] \times \mathbb{R}^{d}\right)}+ & \|w\|_{L_{t}^{\infty} L_{x}^{2}\left(\left[t_{0}, t_{0}+\delta\right] \times \mathbb{R}^{d}\right)} \\
\lesssim & \left\|w\left(t_{0}\right)\right\|_{L^{2}\left(\mathbb{R}^{d}\right)}+\left(\|w\|_{L_{t}^{2} L_{x}^{\frac{d p}{2-p}}\left(\left[t_{0}, t_{0}+\delta\right] \times \mathbb{R}^{d}\right)}^{p}+\|v\|_{L_{t}^{2} L_{x}^{\frac{d p}{2-p}}\left(\left[t_{0}, t_{0}+\delta\right] \times \mathbb{R}^{d}\right)}^{p}\right) \\
& \cdot\left(\|w\|_{L_{t}^{2} L_{x}^{\frac{2 d}{d-2}}\left(\left[t_{0}, t_{0}+\delta\right] \times \mathbb{R}^{d}\right)}+\|v\|_{L_{t}^{2} L_{x}^{\frac{2 d}{d-2}}\left(\left[t_{0}, t_{0}+\delta\right] \times \mathbb{R}^{d}\right)}\right) .
\end{aligned}
$$

By the interpolation and Hölder inequality in time, we have that

$$
\|w\|_{L_{t}^{2} L_{x}^{\frac{d p}{2-p}}\left(\left[t_{0}, t_{0}+\delta\right] \times \mathbb{R}^{d}\right)} \lesssim \delta^{-\frac{s_{c}}{2}}\|w\|_{L_{t}^{2} L_{x}^{\frac{2 d}{d-2}}\left(\left[t_{0}, t_{0}+\delta\right] \times \mathbb{R}^{d}\right)}^{1+s_{c}}\|w\|_{L_{t}^{\infty} L_{x}^{2}\left(\left[t_{0}, t_{0}+\delta\right] \times \mathbb{R}^{d}\right)}^{-s_{c}} .
$$

Moreover, by Proposition 4.6 and Hölder inequality in time, we have that

$$
\|v\|_{L_{t}^{2} L_{x}^{\frac{d p}{2-p}}\left(\left[t_{0}, t_{0}+\delta\right] \times \mathbb{R}^{d}\right)}+\|v\|_{L_{t}^{2} L_{x}^{\frac{2 d}{d-2}}\left(\left[t_{0}, t_{0}+\delta\right] \times \mathbb{R}^{d}\right)} \lesssim \delta^{\frac{1}{2}}
$$


Therefore, by Cauchy-Schwartz's inequality, we obtain that

$$
\begin{aligned}
\|w\|_{L_{t}^{2} L_{x}^{\frac{2 d}{d-2}}\left(\left[t_{0}, t_{0}+\delta\right] \times \mathbb{R}^{d}\right)} & \|w\|_{L_{t}^{\infty} L_{x}^{2}\left(\left[t_{0}, t_{0}+\delta\right] \times \mathbb{R}^{d}\right)} \\
\lesssim 1 & \left\|w\left(t_{0}\right)\right\|_{L^{2}\left(\mathbb{R}^{d}\right)}+\delta^{-\frac{s_{c}}{2}}\left(\|w\|_{L_{t}^{\infty} L_{x}^{2}\left(\left[t_{0}, t_{0}+\delta\right] \times \mathbb{R}^{d}\right)}\right. \\
& \left.+\|w\|_{L_{t}^{2} L_{x}^{\frac{2 d}{d-2}}\left(\left[t_{0}, t_{0}+\delta\right] \times \mathbb{R}^{d}\right)}+\|w\|_{L_{t}^{2} L_{x}^{\frac{2 d}{d-2}}\left(\left[t_{0}, t_{0}+\delta\right] \times \mathbb{R}^{d}\right)}^{p+1}\right) .
\end{aligned}
$$

Choosing $\delta=\delta\left(\left\|w\left(t_{0}\right)\right\|_{L^{2}\left(\mathbb{R}^{d}\right)}\right)>0$ suitably small and the bootstrap, we obtain

$$
\|w\|_{L_{t}^{2} \frac{2 d}{d-2}\left(\left[t_{0}, t_{0}+\delta\right] \times \mathbb{R}^{d}\right)}+\|w\|_{L_{t}^{\infty} L_{x}^{2}\left(\left[t_{0}, t_{0}+\delta\right] \times \mathbb{R}^{d}\right)} \lesssim 1+\left\|w\left(t_{0}\right)\right\|_{L^{2}\left(\mathbb{R}^{d}\right)} .
$$

From Proposition 5.2, $\left\|w\left(t_{0}\right)\right\|_{L^{2}\left(\mathbb{R}^{d}\right)}$ is only dependent on $N$, not dependent on $t_{0}$. Hence $\delta=\delta(N)$. This extends the lifespan to $\mathbb{R}$ and thus proves the global well-posedness.

Lastly, we prove that $u(t) \in \dot{H}^{s_{c}}\left(\mathbb{R}^{d}\right)$ for any $t \in \mathbb{R}$. To this end, we first claim that for any $t_{0} \in \mathbb{R}$,

$$
\|u\|_{L_{t}^{2} L_{x}^{\frac{d p}{2-p}}\left(\left[t_{0}, t_{0}+\delta\right] \times \mathbb{R}^{d}\right)} \lesssim_{N} 1 .
$$

Since $u=v+w$, it reduces to show

$$
\|v\|_{L_{t}^{2} L_{x}^{\frac{d p}{2-p}}\left(\left[t_{0}, t_{0}+\delta\right] \times \mathbb{R}^{d}\right)} \lesssim_{N} 1 ; \quad\|w\|_{L_{t}^{2} L_{x}^{\frac{d p}{2-p}}\left(\left[t_{0}, t_{0}+\delta\right] \times \mathbb{R}^{d}\right)} \lesssim_{N} 1 .
$$

The first one is followed directly by Lemma 4.6. The second one is followed from (5.7), (5.8) and the $L^{2}$ uniform boundedness from Proposition 5.2. This gives (5.9).

Fixing $t_{0} \in \mathbb{R}$, suppose that $w\left(t_{0}\right) \in \dot{H}^{s_{c}}\left(\mathbb{R}^{d}\right)$, then arguing similarly as the proof of Lemma 4.1, we obtain that for some constants $C_{1}, C_{2}>0$,

$$
\begin{aligned}
\|u(t)\|_{L_{t}^{\infty} \dot{H}_{x}^{s c}\left(\left[t_{0}, t_{0}+\delta\right] \times \mathbb{R}^{d}\right)} & \leq\left\|u\left(t_{0}\right)\right\|_{\dot{H}^{s_{c}}\left(\mathbb{R}^{d}\right)}+C_{1}\|u\|_{L_{t}^{2} L_{x}^{\frac{d p}{2-p}}\left(\left[t_{0}, t_{0}+\delta\right] \times \mathbb{R}^{d}\right)}^{p+1} \\
& \leq\left\|u\left(t_{0}\right)\right\|_{\dot{H}^{s_{c}}\left(\mathbb{R}^{d}\right)}+C_{2} .
\end{aligned}
$$

Since $u_{0} \in \dot{H}^{s_{c}}\left(\mathbb{R}^{d}\right)$ and $\delta \sim_{N} 1$, by iteration, we have that for any $t>0$,

$$
\|u(t)\|_{\dot{H}^{s_{c}\left(\mathbb{R}^{d}\right)}} \lesssim_{N} 1+t
$$

The negative direction can be treated similarly. This finishes the proof of Theorem 1.1.

\section{ACKNowledgements}

Part of this work was done while M. Beceanu, A. Soffer and Y. Wu were visiting CCNU (C.C.Normal University) Wuhan, China. The authors thank the institutions for their hospitality and the support. M.B. is partially supported by NSF grant DMS 1700293. Q.D. is supported by NSFC 11971191 and partially by NSFC 11771165. A.S is partially supported by NSF grant DMS 01600749 and NSFC 11671163. Y.W. is partially supported by NSFC 11771325 and 11571118. A.S. and Y.W. would like to thank Ch. Miao for useful discussions. 


\section{REFERENCES}

[1] J. Bourgain, Periodic nonlinear Schrödinger equation and invariant measures. Comm. Math. Phys. 166 (1994), no. 1, 1-26.

[2] J. Bourgain, Invariant measures for the 2D-defocusing nonlinear Schrödinger equation. Comm. Math. Phys. 176 (1996), no. 2, 421-445.

[3] J. Bourgain, Global wellposedness of defocusing critical nonlinear Schrödinger equation in the radial case. J. Amer. Math. Soc. 12 (1999), 145-171.

[4] J. Bourgain and D. Li. On an endpoint Kato-Ponce inequality. Differential Integral Equations 27 (2014), no. 11-12, 1037-1072.

[5] A. Bulut, The defousing energy-supercritical cubic nonlinear wave equation in dimension five. Preprint, arXiv: 1112.0629 .

[6] N. Burq, and N. Tzvetkov, Random data Cauchy theory for supercritical wave equations. I. Local theory. Invent. Math. 173 (2008), no. 3, 449-475.

[7] N. Burq, and N. Tzvetkov, Random data Cauchy theory for supercritical wave equations. II. A global existence result. Invent. Math. 173 (2008), 477-496.

[8] N. Burq, and N. Tzvetkov, Probabilistic well-posedness for the cubic wave equation. J. Eur. Math. Soc. 16 (2014), no. 1, 1-30.

[9] T. Cazenave, Semilinear Schrödinger Equations. Courant Lecture Notes in Mathematics, 10, American Mathematical Society, 2003.

[10] T. Cazenave and F. Weissler, The Cauchy problem for the critical nonlinear Schrödinger equation in $H^{s}$. Nonlinear Anal., Theory, Methods and Applications, 14 (1990), 807-836.

[11] Y. Cho, S. Lee, Strichartz estimates in spherical coordinates. Indiana Univ. Math. J., 62 (2013), no.3, 991-1020.

[12] M. Christ, J. Colliander, and T. Tao, Ill-posedness for nonlinear Schrödinger and wave equations. Preprint arXiv:math/0311048

[13] J. Colliander, M. Keel, G. Staffilani, T. Takaoka, and T. Tao, Global well-posedness and scattering for the energy-critical nonlinear Schrödinger equation in $\mathbb{R}^{3}$, Ann. of Math. (2) 167 (2008), no. 3, 767-865.

[14] J. Colliander, and T. Oh, Almost sure well-posedness of the cubic nonlinear Schrödinger equation below $L^{2}(T)$. Duke Math. J. 161 (2012), no. 3, 367-414.

[15] Y. Deng, Two-dimensional nonlinear Schrödinger equation with random radial data. Anal. PDE 5 (2012), no. 5, 913-960.

[16] Y. Deng, N. Tzvetkov, and N. Visciglia, Invariant measures and long time behaviour for the BenjaminOno equation III. Comm. Math. Phys. 339 (2015), no. 3, 815-857.

[17] B. Dodson, Global well-posedness and scattering for the defocusing, $L^{2}$-critical nonlinear Schrödinger equation when $d \geq 3$. J. Amer. Math. Soc. 25 (2012), no. 2, 429-463.

[18] B. Dodson, Global well-posedness and scattering for the defocusing, $L^{2}$-critical, nonlinear Schrödinger equation when $d=1$. Amer. J. of Math. 138 (2016), no. 2, 531-569.

[19] B. Dodson, Global well-posedness and scattering for the defocusing, $L^{2}$-critical, nonlinear Schrödinger equation when $d=2$. Duke Math. J. 165 (2016), no. 18, 3435-3516.

[20] B. Dodson, Global well-posedness and scattering for the mass critical nonlinear Schrödinger equation with mass below the mass of the ground state. Adv. Math. 285 (2015), 1589-1618.

[21] B. Dodson, Global well-posedness and scattering for the focusing, energy-critical nonlinear Schrödinger problem in dimension $d=4$ for initial data below a ground state threshold. Preprint arXiv:1409.1950.

[22] B. Dodson, J. Lührmann, and D. Mendelson, Almost sure scattering for the $4 D$ energy-critical defocusing nonlinear wave equation with radial data. to appear in Amer. J. Math.

[23] B. Dodson, C. Miao, J. Murphy, and J. Zheng, The defocusing quintic NLS in four space dimensions. Ann. Inst. H. Poincaré Anal. Non Linéaire 34 (2017), no. 3, 759-787.

[24] T. Duyckaerts, J. Holmer, S. Roudenko, Scattering for the non-radial 3D cubic nonlinear Schrödinger equation. Math. Res. Lett. 15 (2008), no. 6, 1233-1250.

[25] T. Duyckaerts, C. Kenig and F. Merle, Scattering for radial, bounded solutions of focusing supercritical wave equations. Int. Math. Res. Not., 2014 (2014), 224-258.

[26] T. Duyckaerts, and T. Roy. Blow-up of the critical Sobolev norm for nonscattering radial solutions of supercritical wave equations on $\mathbb{R}^{3}$. Preprint arXiv:1506.00788 (2015). 
[27] J. Ginibre and G. Velo, Scattering theory in the energy space for a class of nonlinear Schrödinger equations. J. Math. Pures Appl., 64 (1985), 363-401.

[28] Z. Guo and Y. Wang, Improved Strichartz estimates for a class of dispersive equations in the radial case and their applications to nonlinear Schrödinger and wave equations. J. Anal. Math., 124 (2014), 1-38.

[29] M. Keel and T. Tao, Endpoint Strichartz Estimates. Amer. J. Math., 120 (1998), 955-980.

[30] C. E. Kenig and F. Merle, Global well-posedness, scattering and blow-up for the energycritical, focusing, non-linear Schrödinger equation in the radial case. Invent. Math. 166 (2006), 645-675.

[31] C. Kenig, F. Merle, Scattering for $\dot{H}^{\frac{1}{2}}$ bounded solutions to the cubic, defocusing NLS in 3 dimensions. Trans. Amer. Math. Soc. 362 (2010), 1937-1962.

[32] C. Kenig, F. Merle, Nondispersive radial solutions to energy supercritical non-linear wave equations, with applications. Amer. J. Math., 133 (2011), 1029-1065.

[33] C. Kenig, F. Merle, Radial solutions to energy supercritical wave equations in odd dimensions. Discrete Continuous Dynamical Systems-A, 31 (4) (2011), 1365-1381.

[34] C. Kenig, G. Ponce and L. Vega. Well-posedness and scattering results for the generalized Korteweg-de Vries equation via the contraction principle. Comm. Pure Appl. Math., 46 (1993), no. 4, 527-620.

[35] C. E. Kenig, G. Ponce, and L. Vega, On the ill-posedness of some canonical dispersive equations. Duke Math. J., 106 (2001), no. 3, 617-633.

[36] R. Killip, S. Masaki, J. Murphy, and M. Visan. Large data mass-subcritical NLS: critical weighted bounds imply scattering. NoDEA Nonlinear Differential Equations Appl., 24 (2017), no. 4, Art. 38, 33pp.

[37] R. Killip, S. Masaki, J. Murphy, and M. Visan., The radial mass-subcritical NLS in negative order Sobolev spaces. Preprint, arXiv:1804.06753v1.

[38] R. Killip, J. Murphy, and M. Visan., Almost sure scattering for the energy-critical NLS with radial data below $H^{1}\left(\mathbb{R}^{4}\right)$. Prepint, arXiv:1707.09051.

[39] R. Killip, T. Tao, and M. Visan, The cubic nonlinear Schrödinger equation in two dimensions with radial data. J. Eur. Math. Soc. (JEMS) 11 (2009), no. 6, 1203-1258.

[40] R. Killip and M. Visan, Energy-supercritical NLS: critical $\dot{H}^{s}$-bounds imply scattering. Comm. Partial Differential Equations 35 (2010), no. 6, 945-987.

[41] R. Killip and M. Visan, The radial defocusing energy-supercritical nonlinear wave equation in all space dimensions. Prerint, arXiv:1002.1756.

[42] R. Killip and M. Visan, The focusing energy-critical nonlinear Schrödinger equation in dimensions five and higher. Amer. J. Math., 132 (2010), no. 2, 361-424.

[43] R. Killip and M. Visan, Global well-posedness and scattering for the defocusing quintic NLS in three dimensions. Anal. PDE, 5 (2012), no. 4, 855-885.

[44] R. Killip and M. Visan, Nonlinear Schrödinger equations at critical regularity. Clay Math. Proc., 17 (2013), 325-437.

[45] R. Killip, M. Visan, and X. Zhang, The mass-critical nonlinear Schrödinger equation with radial data in dimensions three and higher. Anal. PDE, 1 (2008), no. 2, 229-266.

[46] D. Li, On Kato-Ponce and fractional Leibniz. To appear in Rev. Mat. Iberoam., 2017.

[47] D. Li, X. Zhang, Regularity of almost periodic modulo scaling solutions for mass-critical NLS and applications. Anal. PDE, 3 (2010), no 2, 175-195.

[48] C. Miao, J. Murphy and J. Zheng, The defocusing energy-supercritical NLS in four space dimensions. J. Funct. Anal., 267 (2014), no. 6, 1662-1724.

[49] C. Miao, Y. Wu and X. Zhang, The defocusing energy-supercritical nonlinear wave equation when $d=4$. Preprint.

[50] J. Murphy, Intercritical NLS: critical $\dot{H}^{s}$-bounds imply scattering. SIAM J. Math. Anal., 46 (2014), no. 1, 939-997.

[51] J. Murphy, The radial defocusing nonlinear Schrödinger equation in three space dimensions. Comm. Partial Differential Equations, 40 (2015), no. 2, 265-308.

[52] J. Lürmann, and D. Mendelson, Random data Cauchy theory for nonlinear wave equations of power-type on $\mathbb{R}^{3}$. Comm. Partial Differential Equations, 39 (2014), no. 12, 2262-2283.

[53] A. Nahmod, T. Oh, L. Rey-Bellet, and G. Staffilani, Invariant weighted Wiener measures and almost sure global well-posedness for the periodic derivative NLS. J. Eur. Math. Soc., 14 (2012), no. 4, 12751330.

[54] A. Nahmod, and G. Staffilani, Almost sure well-posedness for the periodic 3D quintic nonlinear Schröinger equation below the energy space. J. Eur. Math. Soc., 17 (2015), no. 7, 1687-1759. 
[55] T. Oh, M. Okamoto, and O. Pocovnicu, On the probabilistic well-posedness of the nonlinear Schröinger equations with non-algebraic nonlinearities. Preprint, arXiv:1708.01568.

[56] T. Oh, and O. Pocovnicu, Probabilistic global well-posedness of the energy-critical defocusing quintic nonlinear wave equation on $\mathbb{R}^{3}$. J. Math. Pures Appl., 105 (2016), 342-366.

[57] O. Pocovnicu, Almost sure global well-posedness for the energy-critical defocusing nonlinear wave equation on $\mathbb{R}^{d}, d=4$ and 5. J. Eur. Math. Soc., 19 (2017), no. 8, 2521-2575.

[58] A. Poiret, D. Robert, and L. Thomann, Probabilistic global well-posedness for the supercritical nonlinear harmonic oscillator. Anal. PDE, 7 (2014), no. 4, 997-1026.

[59] E. Ryckman and M. Visan, Global well-posedness and scattering for the defocusing energy-critical nonlinear Schrödinger equation in $\mathbb{R}^{1+4}$. American Journal of Mathematics, vol. 129, (1), pp. 1-60, 2007.

[60] S. Shao, Sharp linear and bilinear restriction estimates for paraboloids in the cylindrically symmetric case. Rev. Mat. Iberoam., 25 (2009), 1127-1168.

[61] T. Tao, Global well-posedness and scattering for the higher-dimensional energy-critical nonlinear Schrödinger equation for radial data. New York J. Math., 11 (2005), 57-80.

[62] T. Tao, M. Visan, X. Zhang, Global well-posedness and scattering for the defocusing mass-critical nonlinear Schrödinger equation for radial data in high dimensions. Duke Math. J., 140 (2007), no. 1, $165-202$.

[63] L. Thomann, Random data Cauchy problem for supercritical Schrödinger equations. Ann. Inst. H. Poincaré Anal. Non Linéaire 26 (2009), no. 6, 2385-2402.

[64] H.Triebel, Theory of function spaces. II. Monographs in Mathematics, 84. Birkhäuser Verlag, Basel, 1992.

[65] M. Visan, The defocusing energy-critical nonlinear Schrödinger equation in higher dimensions. Duke Math. J. 138 (2007), no. 2, 281-374.

[66] M. Visan, Global well-posedness and scattering for the defocusing cubic nonlinear Schrödinger equation in four dimensions. Int. Math. Res. Not., 2012, no. 5, 1037-1067.

[67] J. Xie and D. Fang, Global well-posedness and scattering for the defocusing $\dot{H}^{s}$-critical NLS. Chin. Ann. Math. Ser. B, 34 (2013), no. 6, 801-842.

Department of Mathematics and Statistics, University at Albany SUnY, Earth Science 110, Albany, NY, 12222, USA,

Email address: mbeceanu@albany.edu

Department of Mathematics, Hubei Key Laboratory of Mathematical Science, Central China Normal University, Wuhan 430079, China.,

Email address: dengq@mail.ccnu.edu.cn

Department of Mathematics, Rutgers University, 110 Frelinghuysen Rd., Piscataway, NJ, 08854, USA,

Email address: soffer@math.rutgers.edu

Center for Applied Mathematics, Tianjin University, Tianjin 300072, China

Email address: yerfmath@gmail.com 